\title{
Análisis hidrogeoquímico-multivariado del agua subterránea del sistema acuífero del Valle Medio del Magdalena, Colombia: Estudio a escala regional
}

\author{
Hydrogeochemical-multivariate analysis of groundwater in the aquifer system of the middle \\ Magdalena valley, Colombia: Regional-scale study
}

Juan Pablo Malagón ${ }^{1, *}$, Adriana Piña ${ }^{1}$, Sebastián Argüello ${ }^{1}$, Leonardo David Donado ${ }^{1}$

${ }^{1}$ Grupo de Investigación Hidrodinámica del Medio Natural, Universidad Nacional de Colombia, Av. NQS 45-03, Laboratorio de Hidráulica (409) Of. 310, Bogotá D.C., Colombia.

* Autor para correspondencia: (J.P. Malagón) jupmalagonna@unal.edu.co

Cómo citar este artículo:

Malagón, J.P., Piña, A., Argüello, S., Donado, L.D., 2021, Análisis hidrogeoquímicomultivariado del agua subterránea del sistema acuífero del Valle Medio del Magdalena, Colombia: Estudio a escala regional: Boletín de la Sociedad Geológica Mexicana, 73 (3), A070421. http://dx.doi.org/10.18268/ BSGM2021v73n3a070421

Manuscrito recibido: 28 de Abril de 2020 Manuscrito corregido: 3 de Abril de 2021 Manuscrito aceptado: 12 de Abril de 202

La revisión por pares es responsabilidad de la Universidad Nacional Autónoma de México.

Este es un artículo Open Access bajo la licencia CC 1 -NCSA (https://creativecommons.org/licenses/by-nc-sa/4.0/)

\section{RESUMEN}

Para identificar los principales procesos geoquímicos que determinan la composición química y la calidad del agua subterránea del Sistema Acuífero del Valle Medio del Magdalena Colombia (SAVMM), se creó una base de datos con las caracterizaciones fisicoquímicas disponibles en las diferentes entidades gubernamentales y privadas ubicadas en el área de estudio. En total se recopilaron 2207 caracterizadores fisicoquímicas para el periodo comprendidos entre los años 2005 a 2014. Para este estudio se conservaron las muestras que estuvieran completas, no tuvieran errores de transcripción y que tuvieran un error analítico menor o igual a +/- $10 \%$. El número de caracterizaciones que resultaron válidas fueron 140. Se utilizaron métodos gráficos, hidroquímicos y estadísticos, para la comprensión de los procesos geoquímicos. De acuerdo con el análisis del diagrama de Piper se observó que el tipo de agua bicarbonatada cálcica es la facie hidrogeoquímica dominante a lo largo del área de estudio, seguida por la del tipo bicarbonatada sódica y clorurada sódica. El estudio se complementó haciendo un análisis estadístico multivariado, incluyendo un Análisis de Componentes Principales (ACP) y Análisis de Agrupamiento Jerárquico (AAJ). Con el ACP, se determinaron tres componentes principales que representan el 59.4\% de la variabilidad de los datos y con el AAJ se obtuvieron 6 grupos que representan las 140 caracterizaciones fisicoquímicas. La geología e hidrogeología del área de estudio se correlacionó con los resultados del ACP y del AAJ, identificando así 3 procesos geoquímicos principales que determinan la química del agua subterránea en el SAVMM: 1) Recarga - infiltración de agua meteórica, 2) Disolución interacción del agua con silicatos y carbonatos 3 ) Alteración Antropogénica - contaminación por el desarrollo de actividades industriales realizadas en el área de estudio.

Palabras clave: Hidrogeoquímica, Análisis de Componentes Principales, Análisis de Agrupamiento Jerárquico, Procesos Geoquímicos.

\section{ABSTRACT}

To identify the main geochemical processes that determine the chemical composition and quality of the groundwater of the Aquifer System of the Middle Valley of Magdalena - Colombia (SAVMM), a database was created with the physicochemical characterizations available in the different governmental and private entities located in the study area. In total, 2207 data were collected for the period between the years 2005 to 2014. For this study, samples that were complete, did not have transcription errors, and had an analytical error less than or equal to $+/-10 \%$ were kept. The number of valid samples was 140. Graphic, hydrochemical and statistical methods were used to understand geochemical processes. According to the analysis of the Piper diagram, it was observed that the calcium bicarbonate type of water is the dominant hydrogeochemical face throughout the study area, followed by the sodium bicarbonate and sodium chloride type. The study was complemented by doing a multivariate statistical analysis, including a Principal Component Analysis (PCA) and Hierarchical Cluster Analysis $(A A J)$. With the PCA, three components that represent $59.4 \%$ of the variability of the data were determined and with the AJJ 6 groups were obtained that represent the 140 physicochemical characterizations. The geology and hydrogeology of the study area was correlated with the results of the $A C P$ and the AAJ, thus identifying 3 main geochemical processes that determine the chemistry of groundwater in the SAVMM: 1) Recharge - meteoric water infiltration, 2) Dissolution - interaction of water with silicates and carbonates 3) Anthropogenic Alteration - contamination due to the development of industrial activities carried out in the study area.

\begin{tabular}{|c|c|c|}
\hline Keyzords: & \multicolumn{2}{|c|}{ Hydrogeochemistry, } \\
\hline Principal & Component & Analysis, \\
\hline $\begin{array}{l}\text { Hierarchical } \\
\text { Geochemical }\end{array}$ & $\begin{array}{l}\text { Clustering } \\
\text { rocesses. }\end{array}$ & Analysis, \\
\hline
\end{tabular}




\section{Introducción}

Desde principios de la década de 1920, se han desarrollado diversas técnicas gráficas y estadísticas para facilitar el entendimiento geoquímico del agua subterránea, con el objetivo final de dividirla en grupos homogéneos (Güler et al., 2002). Las técnicas gráficas más conocidas y empleadas son los diagramas de Piper (1944), Stiff (1951), Gibbs (1970) y Mifflin (1988). Sin embargo, para la evaluación de gran cantidad de datos, los métodos estadísticos multivariados facilitan el análisis, por lo que, han sido utilizados ampliamente para investigar fenómenos ambientales (Gong et al., 2005; Chou et al., 2012; Giménez et al., 2017). Dentro de los métodos más utilizados se tiene el análisis estadístico multivariado (AEM) el cual, es una herramienta implementada para el análisis del agua subterránea que permite la definición de grupos distintivos, así como correlaciones entre sus parámetros (Cloutier et al., 2008). El Análisis de Componentes Principales (ACP) es una técnica estadística utilizada para descifrar patrones dentro de grandes conjuntos de datos (Farnham et al., 2003) y el Análisis de Agrupamiento Jerárquico (AAJ) permite identificar firmas químicas similares entre las muestras y así agruparlas en grupos denominados clústers (Everitt et al., 2011).

El AEM ha sido utilizado, por ejemplo, por Steinhorst y Williams (1985) para identificar fuentes de agua subterránea y por Farnham et al., (2003) para identificar los procesos relacionados con los elementos traza. Por otra parte, el ACP ha sido implementado para la caracterización de acuíferos locales, Melloul y Collin (1992) identificaron los principales grupos de agua y componentes que afectan su calidad en el acuífero de la llanura costera de Israel. Villegas et al., (2013) evaluaron los principales procesos que controlan la química del agua subterránea en el acuífero confinado de Urabá-Colombia; Matiatos et al. (2014) apoyaron su estudio isotópico con un ACP con el fin de interpretar las variaciones espaciales en la calidad del agua subterránea en la región del Peloponeso nororiental, Grecia. A su vez, Cloutier, et al. (2008), Montcoudiol et al., (2014) y Ghesquière et al.,(2015), utilizaron en sus estudios el AAJ, para identificar los procesos primarios responsables de la química y evolución geoquímica del agua subterránea en la región de Outaouais (Canadá). Recientemente, Blake et al., (2016) evaluaron la procedencia del agua termal ubicada en la cuenca carbonífera de Dublín (Irlanda), Noshadi y Ghafourian (2016) investigaron la calidad del agua de la provincia de Fars, ubicada al sur de Irán, en ambos trabajos utilizaron tanto el AAJ como el ACP.

Como caso de estudio se presenta la región del sistema acuífero del Valle Medio del Magdalena Colombia (SAVMM), que se encuentra ubicada en la cuenca hidrográfica del Magdalena, Cauca, donde se registra el mayor consumo de agua subterránea en el pais, con un 78\% del volumen concesionado a nivel nacional (IDEAM, 2014a). Los principales usos que se le da al agua subterránea en esta zona están referidos, al suministro de agua potable, el desarrollo de actividades agroindustriales y la explotación minera e hidrocarburífera, lo que representa una fuente potencial de alteración de la calidad del agua subterránea (IDEAM, 2014a). Adicionalmente, el área de estudio se encuentra ubicada en una zona con gran potencial para la extracción de gas de lutita (ANH, 2012), para cuya explotación se prevé el uso del Fracturamiento Hidráulico Multietapa con Perforación Horizontal -FHMPH, técnica denominada Fracking (DNP, 2014). Para el uso del (FPPH) en el país se realizarán antes Proyectos Piloto Integrales de Investigación (PPII) (Consejo del Estado, 2018), con el fin de obtener la información suficiente para evaluar la viabilidad económica y ambiental de este tipo de desarrollos. Diferentes estudios asocian el FHMPH, con diversos impactos al recurso hídrico subterráneo, conflictos por su uso y riesgos de contaminación (Osborn et al., 2011; Darraha et al., 2014; U.S.EPA, 2016; Costa et al., 2017).

La cantidad y disponibilidad del agua subterránea en la región ha sido caracterizada gracias a los estudios hidrogeológicos realizados por las autoridades ambientales nacionales y regionales (IDEAM, 2013; 2014a). Sin embargo, el tema 
de calidad, y más concretamente los procesos geoquímicos que controlan la composición química del agua subterránea, están pobremente comprendidos. En el SAVMM se han realizado estudios puntuales que contienen información fisicoquímica para determinar la calidad del agua subterránea y determinar las facies hidrogeoquímicas (INGEOMINAS, 2002; Mejía, 2008). El tipo de agua predominante en el acuífero de los municipios de Puerto Berrío y Puerto Nare (que forman parte del SAVMM) fue determinada por Arismendy et al., (2003) y corresponde a agua del tipo bicarbonatada sódica y en menor proporción bicarbonatada cálcica. En 2013 se delimitó el SAVMM (IDEAM, 2013) y en 2014, el IDEAM presentó un análisis hidrogeológico de la zona, en el cual concluyó que el nivel de conocimiento del SAVMM no es suficiente para la gestión del agua subterránea (IDEAM, 2014a). Actualmente, sólo se han presentado resultados dispersos de informes de consultoría local, aproximaciones generales y puntales de la calidad y clasificación geoquímica, así como análisis fisicoquímicos dispersos de la calidad del agua subterránea, pero a la fecha no se han realizado estudios exhaustivos que incluyan el uso de técnicas estadísticas multivariadas para identificar y comprender los procesos geoquímicos que controlan la composición química del agua subterránea en el SAVMM. Por esta razón, con el fin de suplir esta deficiencia en el conocimiento hidrogeológico del SAVMM, en este trabajo se compilaron las caracterizaciones fisicoquímicas disponibles en los Estudios de Impacto Ambiental (EIA), Planes de Manejo Ambiental (PMA) e Informes de Cumplimiento Ambiental (ICA), presentados ante la Autoridad Nacional de Licencias Ambientales (ANLA), y por parte de las empresas interesadas en desarrollar proyectos de exploración y producción de hidrocarburos en el área de estudio.

En este contexto se busca analizar las características hidrogeoquímicas del Sistema Acuífero del Valle Medio del Magdalena (SAVMM), Colombia, con el fin de comprender los datos químicos y procesos geoquímicos que determi- nan la composición del agua subterránea, y así aumentar el conocimiento para la gestión de este recurso natural ante la creciente demanda y riesgo de contaminación.

\section{2. Área de estudio}

El SAVMM se encuentra ubicado en la cuenca sedimentaria del Valle Medio del Magdalena, abarca una superficie de $14913 \mathrm{~km}^{2}$ y se encuentra limitado por la Cordillera Central al occidente y la Cordillera Oriental al oriente como se aprecia en la figura 1. El SAVMM se caracteriza por tener una topografía suave, con una elevación promedio de 186 msnm y máxima de 600 msnm. La dinámica fluvial de la zona es contralada por el Río

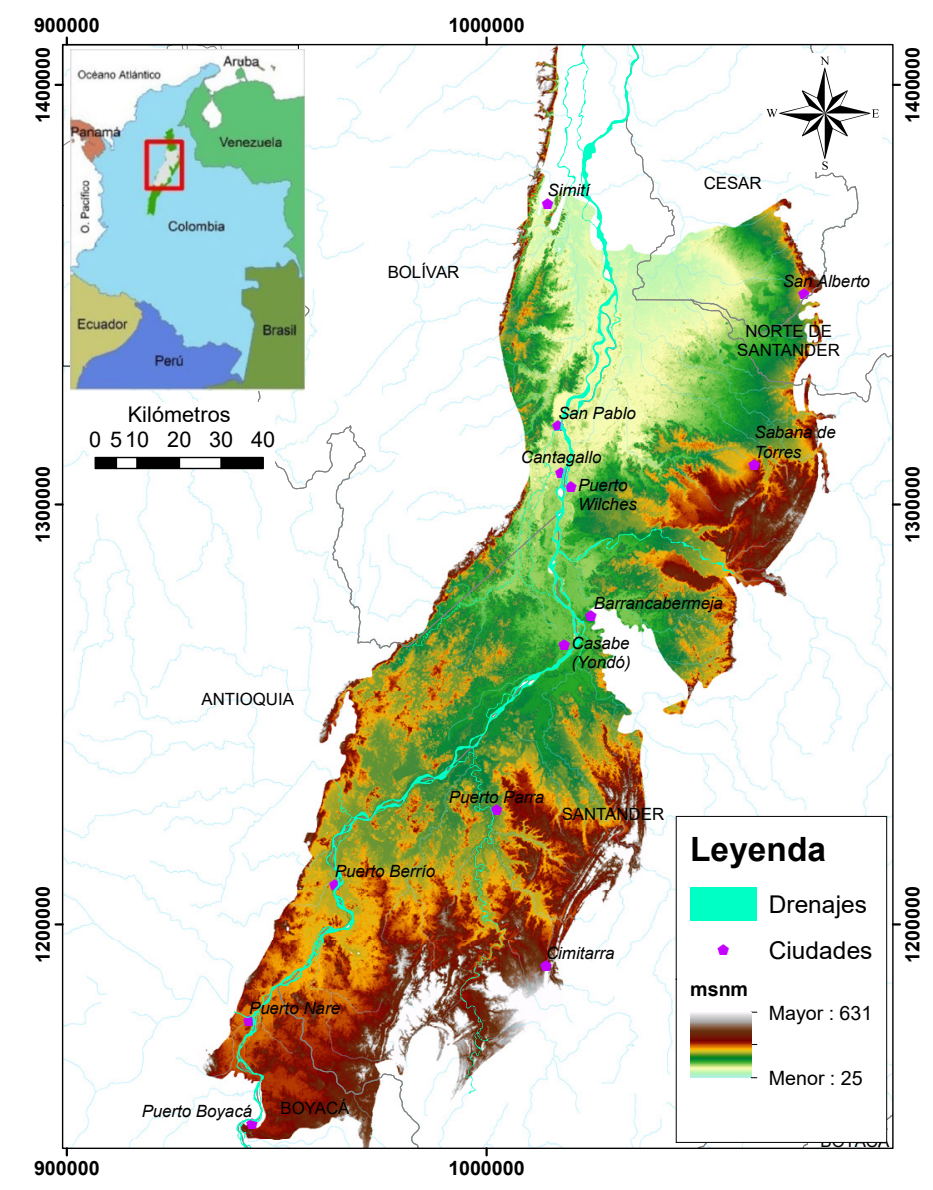

Figura 1 Mapa de localización geográfica del Sistema Acuífero del Valle Medio del Magdalena, en el cual se resalta la topografía en msnm y se presentan los principales drenajes (Escala 1:980000 - modificado de IDEAM 2014). 
Magdalena, principal corriente hídrica superficial del país. La zona de estudio tiene una temperatura media de $25.6^{\circ} \mathrm{C}$, un régimen de precipitación bimodal, registrando precipitaciones medias de $2871 \mathrm{~mm} /$ año, una evapotranspiración potencial calculada mediante el método de FAO-Pennman Monthieth (Allen et al., 1998) de 1313 mm/año, registrando los mayores déficits hídricos en la zona norte del área de estudio.

\subsection{GEOLOGÍA}

El SAVMM se encuentra delimitado por diferentes sistemas de fallas, cinturones plegados y basamento compuesto de unidades calcáreas intercaladas con unidades de ambientes marinos profundos de edad cretácica de la historia geológica colombiana (ANH, 2007). El área de estudio comprende unidades sedimentarias y cristalinas relacionadas con diferentes procesos geológicos desarrollados entre el Jurásico y el Cuaternario reciente. El principal agente que influyo en los procesos de sedimentación de las rocas ubicadas en el SAVMM fue la incursión marina desarrollada durante el Cretácico, que dejó a su paso evidencias paleontológicas en algunas unidades. A partir del Paleógeno, inicia el proceso de inversión tectónica de la cuenca, lo que resulta en un cambio en el ambiente de sedimentación de las unidades litoestratigráficas; las unidades pasan de un ambiente marino a un ambiente controlado por procesos fluviales evidenciados en las formaciones con mayor presencia en el SAVMM: Grupo Real, Formación Mesa y Depósitos Cuaternarios (INGEOMINAS, 2003) como se muestra en la figura 2.

Por encima del basamento calcáreo se encuentran las rocas sedimentarias y depósitos no consoli-

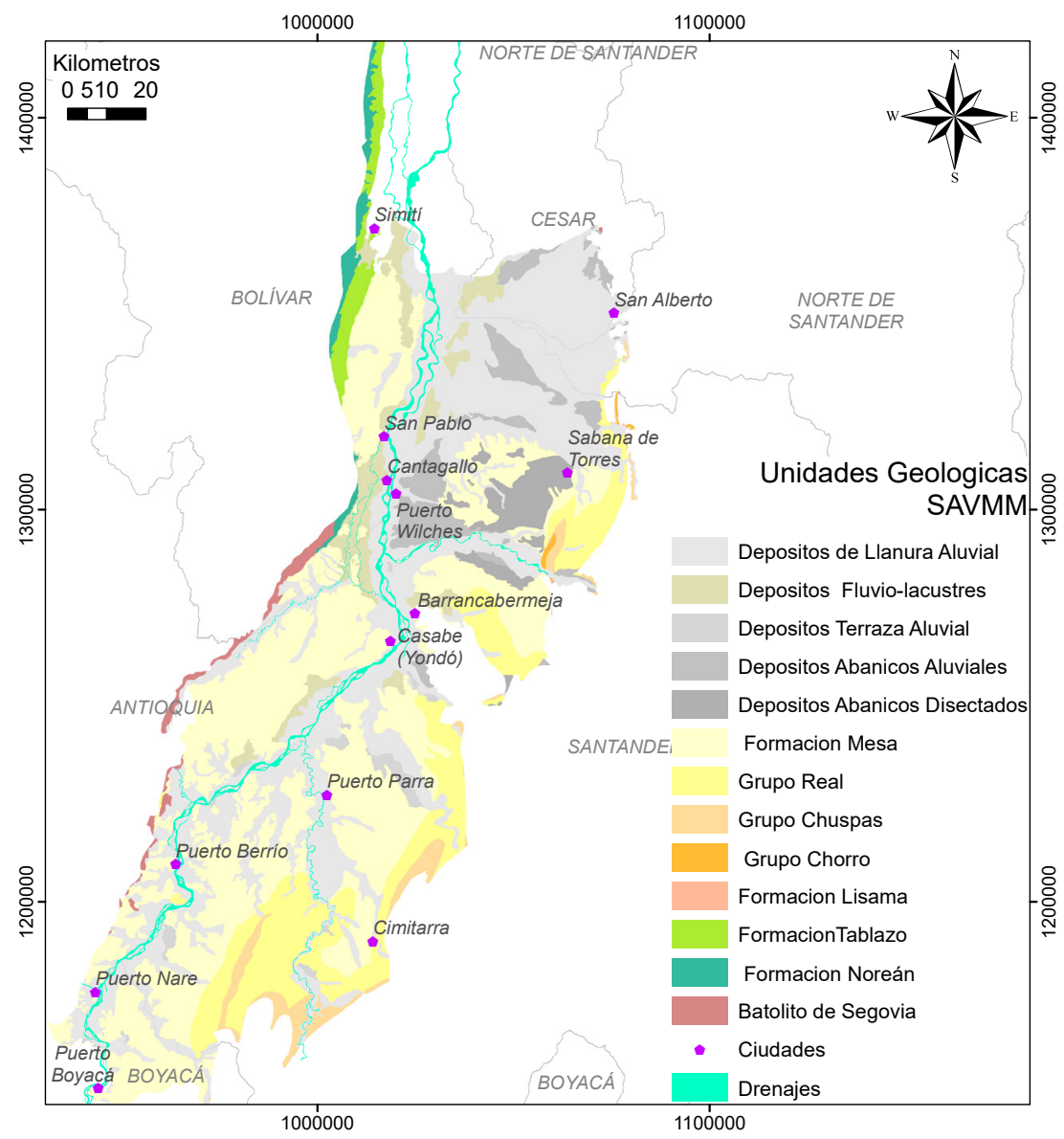

Figura 2 Mapa Geológico del Sistema Acuífero Valle Medio del Magdalena (Escala 1:980000 - modificado de IDEAM 2014). 
dados asociados a la dinámica fluvial de las zonas más profundas de la cuenca del Valle Medio del Magdalena. Debido a esto se dan variaciones granulométricas ideales para el almacenamiento del agua (INGEOMINAS, 2003).

El SAVMM se encuentra cubierto en un 95\% por rocas sedimentarias divididas en 12 formaciones litoestratigráficas. Del mismo modo, se identificaron cuerpos litodémicos, para un total de 16 unidades litológicas en la parte occidental del área de estudio, pertenecientes a procesos tectónicos que dieron origen a la formación de la cordillera central, como son: el Batolito de Segovia y el Complejo Cajamarca. A continuación, se hace una descripción litológica de las unidades con principal interés hidrogeológico siguiendo la secuencia estratigráfica del SAVMM (Figura 3).

\subsubsection{GRETÁCICO}

En la Formación La Luna se caracteriza por su predominancia de lodolitas calcáreas (grises) fosilíferas laminadas. Dentro de su composición mineralógica se resalta la presencia de calcita, dolomita, y fragmentos líticos de silicatos (Morales et al., 1958).

\subsubsection{PALEÓGENO}

\section{Grupo Chorro}

Hace referencia a las Formaciones Esmeraldas y La Paz del Eoceno y Oligoceno temprano. La Formación Esmeraldas fue nombrada por Morales et al. (1958). Ésta se compone de areniscas grises y verdosas de grano fino con intercalaciones de limolitas y lutitas rojas, púrpura y pardas; contiene algunas capas de carbón. Mineralógicamente su composición es principalmente cuarzosa con fragmentos de feldespato potásico alterados localmente a caolinita y otros minerales de arcilla.

La Formación La Paz es la porción basal del grupo Chorro. Sus depósitos son predominantemente de río trenzado y se componen de areniscas de color gris claro y conglomerados lenticulares, con intercalaciones de calizas y dolomitas grises moteadas (Montgomery, 1992). Dada la heterogeneidad de su material parental, mineralógicamente puede contener abundantes silicatos, minerales de arcilla y carbonatos.

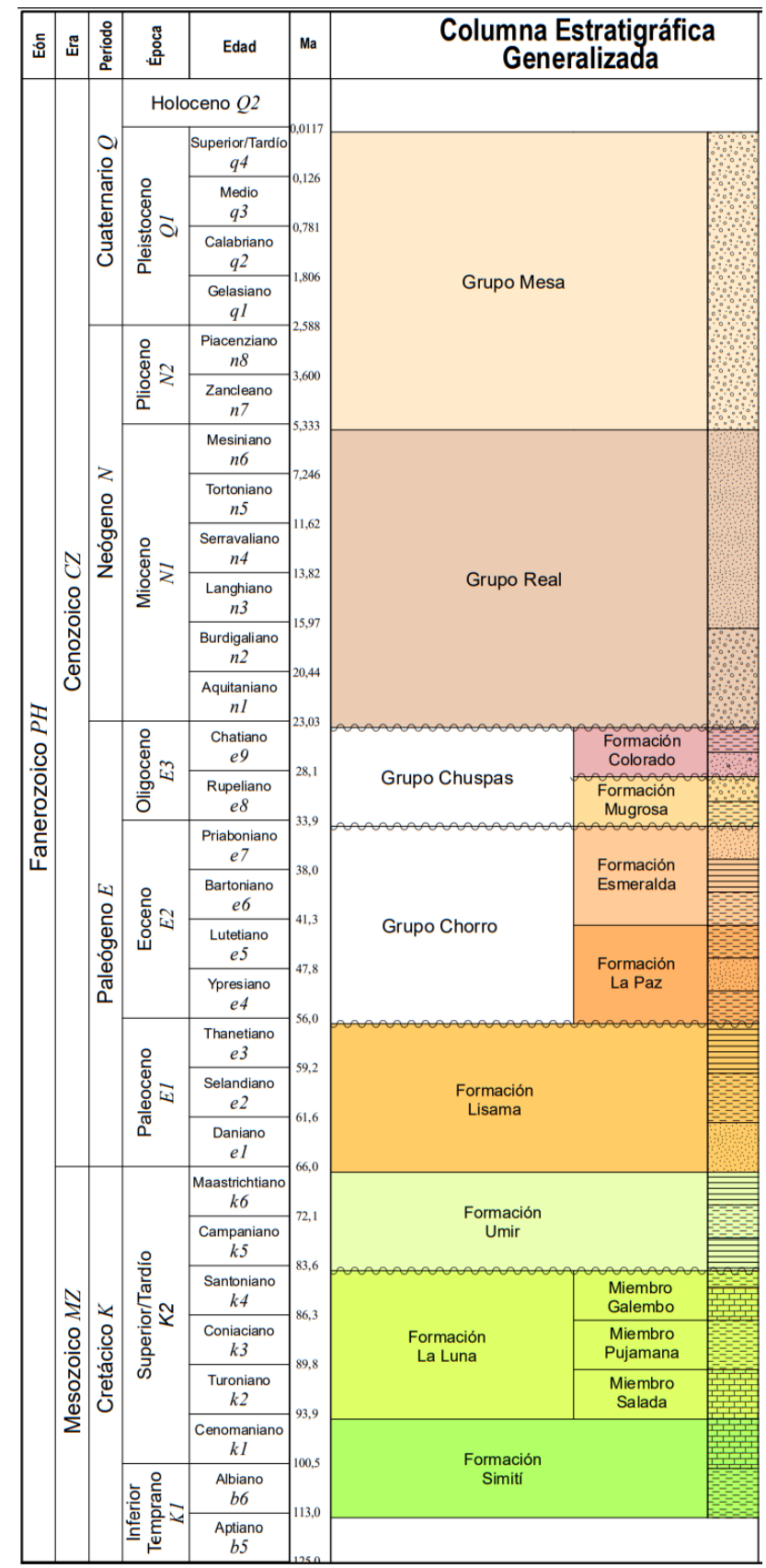

Figura 3 Columna estratigráfica generalizada de la Cuenca del Valle Medio del Magdalena (Tomada de Servicio Geológico Colombiano -SGC, 2019). 
Grupo Chuspas

Corresponde a las formaciones Mugrosa y Colorado (Morales et al., 1958). La parte basal de la formación consiste en areniscas de grano fino a medio de color gris con intercalaciones de lutitas grises y azules (Mora et al., 2004). Al tener un nivel fosilífero de alta competencia se infiere su composición rica en calcita y dolomita biogénica. De acuerdo con las dataciones en los horizontes fosilíferos Los Corros y Mugrosa, la edad de la Formación Mugrosa se encuentra en el intervalo Oligoceno temprano a medio (Taborda, 1965).

La formación Colorado fue formalizada por Morales et al. (1958), el límite inferior de la Formación Colorado es el horizonte fosilífero que se encuentra al tope de la Formación Mugrosa, y el límite superior se marca en el horizonte fosilífero de La Cira, la cual es inconforme con el suprayacente Grupo Real. El espesor total en la sección tipo es de 1,250 metros (Ward et al., 1973). Con base en dataciones relativas se da una edad de Oligoceno Superior a Mioceno Inferior para esta unidad (Ward et al., 1973).

\subsubsection{NEÓGENO}

\section{Grupo Real}

Descrita y publicada originalmente por Porta (1974). En el área tipo, la sucesión inicia con conglomerados compuestos por fragmentos silíceos de chert blanco, arcillolitas ferruginosas y horizontes locales de carbón, seguidos de 500 $\mathrm{m}$ de areniscas conglomeráticas. Se compone de clastos de fragmentos líticos como rocas graníticas de los alrededores del SAVMM. Estas rocas graníticas son fuente de plagioclasas, feldespato potásico, cuarzo y biotitas.

Ascendiendo desde la base se encuentran 1300 $\mathrm{m}$ de lodolitas abigarradas grises a rojizas de origen continental intercaladas con areniscas grises en capas delgadas (De Porta, 1974). Seguidas se encuentran $1100 \mathrm{~m}$ de areniscas conglomeráticas de coloración gris azulosa con contenidos locales de hornblenda y augita (Morales et al., 1958). El ambiente de formacion de esta unidad está asociado a ambientes fluviales de alta energía, donde se produce alteracion de rocas igneas que componen el basamento de la cordillera oriental. Debido a esto, la unidad presenta fragmentos líticos de rocas graníticas ricas en albita, feldespato potásico y filosicatos como muscovita y biotita (Cañas et al., 2019).

\section{Formación Mesa}

La Formación Mesa en el área de estudio se encuentra compuesta por conglomerados masivos, areniscas líticas y menores capas de lodolitas (Morales, 1958). Al abarcar diferentes depósitos de origen fluvial dentro de la cuenca, los clastos de esta unidad tienen composición variable como cuarzo, fosfatos, arcosas o andesitas (Cañas et al., 2019). Mineralógicamente dado su material parental ígneo, puede contener abundante cuarzo, feldespato potásico y plagioclasas (albita) y, en menor medida, filosilicatos (Cañas et al., 2019).

\subsubsection{CUATERNARIO}

Los depósitos cuaternarios son depósitos no consolidados, los cuales, hasta el momento, no han sufrido procesos diagenéticos o de litificación hasta llegar a formar rocas propiamente dichas. El origen principalmente de estos depósitos puede ser aluvial, coluvial, y fluvio-lacustre. Dado su ambiente de formación, se caracterizan por tener alto contenido de cuarzo, feldespato potásico y matriz rica en minerales de arcilla (De Porta, 1974).

\section{Depósitos de terrazas aluviales}

Esta unidad se encuentra conformada por diferentes depósitos de origen fluvial. En algunas localidades predominan arenas líticas y capas gruesas canaliformes de conglomerados clastosoportados, polimícticos, angulares a subredondeados. Estos depósitos presentan una composición limo-arcillosa en la parte superior y de intercalaciones de gravas, arenas gruesas y limos hacia la base, depositados en un ambiente fluvial. El espesor máximo de 40 m sobreyace en la Formación Mesa, depósitos de abanicos y coluviales recientes (Cañas et al., 2019). 
Abanicos aluviales

Los abanicos aluviales se encuentran asociados a la acción erosiva desarrollada en el flanco occidental de la cordillera oriental. Dichos cuerpos están conformados por sedimentos no consolidados compuestos de fragmentos graníticos y andesíticos provenientes de las cordilleras oriental y central. La composición mineralógica está conformada de feldespatos potásicos, cuarzo, plagioclasas y micas (Cañas et al., 2019).

\section{Depósitos fluvio - lacustres}

En el área de estudio se distinguen sedimentos fluvio - lacustres, depósitos de llanura de inundación y de planicie fluvio - lacustre. La característica estratigráfica principal de este tipo de depósitos es de facies de sedimentos finos de arena y lodos con altos contenidos de materia orgánica (Cañas et al., 2019).

\section{Depósitos fluviales del Río Magdalena}

Se refiere a los depósitos de llanura aluvial de los afluentes de Río Magdalena. Se encuentran generalmente, caracterizados por sedimentos tamaño arcillas y limos, aunque se observan aportes en menor cantidad de granos tamaño arena y grava. Estos depósitos se caracterizan por presentar bloques. Los fragmentos transportados por el Rio Magdalena son de origen ígneo y sedimentario. Dentro de los fragmentos ígneos se presentan silicatos como cuarzo y feldespatos; mientras que los fragmentos sedimentarios son ricos en cuarzo, calcita y dolomita provenientes de las calizas de la zona (Cañas et al., 2019).

\subsection{HIDROGEOLOGÍA}

El SAVMM está conformado por distintas unidades hidrogeológicos de edad cuaternaria cuya principal característica es la baja consolidación y predominio de sedimentos como arena y grava, intercalados con materiales de grano fino como arcillas y limos (Cañas et al., 2019). Dentro del SAVMM, las unidades que funcionan como acuíferos son los depósitos aluviales recientes y de terraza que afloran en cercanías del Río
Magdalena cuyo origen se asocia a dicho cuerpo de agua, y los sedimentos detríticos poco consolidados (areniscas, conglomerados) de la Formación Mesa y el Grupo Real. Además de las unidades clásticas, existen unidades hidrogeológicas con porosidad secundaria debido a su composición calcárea como la Formación La Luna. Estas unidades se distribuyen y conforman acuíferos libres a semilibres, multicapa en unidades recientes y acuíferos semiconfinados a confinados en unidades más antiguas del Neógeno (IDEAM, 2013). El SAVMM tiene una recarga directa a partir de la infiltración sobre las unidades hidrogeológicas libres que lo conforman, estimada entre 0 y 500 $\mathrm{mm} / \mathrm{año}$, con espesores saturados que varían de 80 a $230 \mathrm{~m}$ y valores de la transmisividad entre 100 a $200 \mathrm{~m}^{2}$ / día (IDEAM, 2014b).

El régimen hidrogeológico está controlado por el Río Magdalena, siendo éste la principal zona de descarga existente para el área de estudio, debido a la configuración topográfica y geológica del SAVMM. La dirección del flujo regional subterráneo es convergente hacia el Río Magdalena, pero con sentido norte (INGEOMINAS, 2003). Sin embargo, en los períodos donde aumenta la pluviosidad, puede darse una recarga desde el río hacía de las unidades hidrogeológicas adyacentes al Río Magdalena, gracias a la sobresaturación de éstas; mientras que en temporada de estiaje puede darse un proceso contrario donde el agua subterránea almacenada en las unidades adyacentes descarga y aporta al caudal base del río.

\section{Materiales y métodos}

El proceso de investigación consistió en la recopilación de información fisicoquímica disponible en las diferentes entidades gubernamentales y privadas ubicadas en el área de estudio. Posteriormente se verificó la calidad de los datos considerando: i) valores químicos y fisicoquímicos reportados razonables, ii) errores de transcripción y, iii) errores analíticos en el balance iónico menores o iguales al 10\% (Hounslow, 1995). El análisis de los datos se realizó utilizando métodos gráficos, estadísticos 
descriptivos y multivariados (ACP y $\mathrm{AAJ}$ ), que junto con la correlación del conocimiento geológico e hidrogeológico de la zona se utilizó para identificar los principales procesos geoquímicos que determinan la composición química del agua subterránea en el SAVMM.

\subsection{SELEGGIÓN Y DESGRIPGIÓN DE LAS MUESTRAS}

Para este estudio se recopilaron datos de parámetros fisicoquímicos del agua subterránea tomados en pozos, aljibes, manantiales y piezómetros, realizados por parte de las empresas interesadas en desarrollar proyectos de exploración y producción de hidrocarburos en la cuenca del Valle Medio del Magdalena, disponibles en Estudios de Impacto Ambiental, Plan de Manejo Ambiental e Informes de Cumplimiento Ambiental, presentados ante la Autoridad Nacional de Licencias Ambientales y a su vez recopiladas por el Instituto de Hidrología, Meteorología y Estudios Ambientales de Colombia (IDEAM), para el desarrollo del Estudio Nacional del Agua 2014 (IDEAM, 2014a). Con base en el proceso de recopilación, se creó una base de datos hidroquímica en formato Excel del Valle Medio del Magdalena - BDHVMM. En total se recopiló la información de 84 proyectos petroleros licenciados, de los cuales 56 tenían información útil para el desarrollo del presente estudio. La información utilizada para conformar la BDHVMM se presentan en la tabla 1.

El trabajo de procesamiento de la información quedó registrado en el sitio web de libre consulta HidroVisor ASVAMM (Agua Subterránea del Valle Medio del Magdalena) (https://sites.google. com/site/invhidrovmm/ubicacion/home). Esta página de internet fue creada con el fin de compartir la información compilada sobre ASVAMM con las entidades públicas, privadas y el público en general como aporte.

Se recopilaron en total 2207 caracterizaciones fisicoquímicas para el periodo comprendido entre los años 2005 a 2014 incluyendo muestras temporales (muestras recogidas en un período de tiempo diferente en el mismo lugar). Posterior al análisis de calidad, descrito en la siguiente sección, la base de datos quedó finalmente conformada por 140 caracterizaciones. En el caso de múltiples muestras pertenecientes a la misma localización, los datos de las muestras más recientes o completas fueron las consideradas para el análisis estadístico.

Para el análisis estadístico de la información se utilizaron 12 parámetros: Conductividad Eléctrica (CE), Sólidos Disueltos Totales (SDT), pH, Calcio - $\mathrm{Ca}^{2+}$, Sodio $-\mathrm{Na}^{+}$, Potasio $-\mathrm{K}^{+}$, Magnesio $-\mathrm{Mg}^{2+}$, Hierro Total - Fe, Cloruros - Cl-, Sulfatos - $\mathrm{SO}_{4}^{2-}$, Bicarbonatos - $\mathrm{HCO}_{3}^{-}$y Nitratos - $\mathrm{NO}_{3}^{-}$. Estos parámetros proveen información útil acerca de los procesos que controlan el agua subterránea en el área de estudio (Güler et al., 2002). Se consideró a la CE y los SDT como parámetros suplementarios, dado que, de alguna forma, resumen la información del resto de parámetros y su valor indica en cierta medida la concentración de los iones mayoritarios $\left(\mathrm{Ca}^{2+}, \mathrm{Na}^{+}, \mathrm{K}^{+}, \mathrm{Mg}^{2+}\right.$, Fe total, $\mathrm{Cl}-, \mathrm{SO}_{4}^{2-}, \mathrm{HCO}_{3}^{-}$y $\mathrm{NO}_{3}$ ), lo que puede generar que el método concentre toda su atención en ellos y dificulte la identificación de relaciones relevantes sobre el resto de parámetros (Güler et al., 2002).

\subsection{ANÁLISIS DE GALIDAD DE DATOS}

En el presente estudio se consideró que los datos cuentan con el aseguramiento y control de calidad en el momento de la colecta de la muestra y análisis. Sin embargo, se examinaron los datos para verificar que las caracterizaciones compiladas contaban con la calidad pertinente para el desarrollo de esta investigación. Durante esta revisión de datos, se evidenciaron errores como: muestras con ubicaciones inciertas o con coordenadas invertidas, sin registro de profundidades, unidades de medida inconsistentes (todos los valores de concentración se convirtieron a un formato $\mathrm{mg} / \mathrm{L}$ ), y valores atípicos o caracterizaciones incompletas para las 12 variables consideradas. Los valores incompletos pueden hacer imposible el uso de técnicas gráficas para el análisis de la química del agua o limitar la calidad del análisis estadístico (Güler et al., 2002). Por tal razón se emplearon relaciones químicas para estimar los valores de datos faltantes (Fagundo et al., 2005). La CE se calculó a partir de 
Tabla 1. Información utilizada para conformar la base de datos hidroquímicos del Valle Medio del Magdalena BDHVMM. Fuente de datos: ANLA e IDEAM.

\begin{tabular}{|c|c|c|c|c|}
\hline \multirow[b]{2}{*}{ Código } & \multirow[b]{2}{*}{$\begin{array}{c}\text { No de } \\
\text { Expediente } \\
\text { ANLA }\end{array}$} & \multicolumn{3}{|c|}{ Numero de muestras } \\
\hline & & $\begin{array}{l}\text { Número de } \\
\text { puntos de } \\
\text { Agua }\end{array}$ & $\begin{array}{l}\text { Número de } \\
\text { caracterizaciones } \\
\text { por punto de } \\
\text { agua }\end{array}$ & $\begin{array}{c}\text { Número de } \\
\text { caracterizaciones } \\
\text { que cumplen los } \\
\text { estándares de } \\
\text { calidad }\end{array}$ \\
\hline 1 & LAM0037 & 192 & 29 & 25 \\
\hline 2 & LAM0040 & 39 & 7 & \\
\hline 3 & LAM0180 & 153 & 455 & 16 \\
\hline 4 & LAM0203 & 4 & 2 & \\
\hline 5 & LAM0232 & 15 & 15 & - \\
\hline 6 & LAM0242 & 65 & 23 & _ \\
\hline 7 & LAM0332 & 156 & 34 & - \\
\hline 8 & LAM0855 & 497 & 436 & 30 \\
\hline 9 & LAM1009 & 420 & 100 & 1 \\
\hline 10 & LAM1277 & 128 & 25 & _ \\
\hline 11 & LAM1470 & 2 & 2 & _ \\
\hline 12 & LAM1913 & 61 & 95 & $\overline{4}$ \\
\hline 13 & LAM1994 & 65 & 71 & 10 \\
\hline 14 & LAM2199 & 49 & 4 & \\
\hline 15 & LAM2249 & 513 & 353 & 1 \\
\hline 16 & LAM2317 & 178 & 79 & 9 \\
\hline 17 & LAM2762 & 1 & 1 & _ \\
\hline 18 & LAM2903 & 1 & & - \\
\hline 19 & LAM3090 & 44 & 13 & - \\
\hline 20 & LAM3176 & 3 & - & - \\
\hline 21 & LAM3410 & 4 & & - \\
\hline 22 & LAM3435 & 1 & 1 & - \\
\hline 23 & LAM3539 & 4 & - & - \\
\hline 24 & LAM3654 & 2 & & - \\
\hline 25 & LAM3846 & 39 & 3 & - \\
\hline 26 & LAM3856 & 3 & 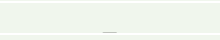 & - \\
\hline 27 & LAM3857 & 9 & 9 & - \\
\hline 28 & LAM3965 & 201 & 181 & 6 \\
\hline 29 & LAM4083 & 11 & & - \\
\hline 30 & LAM4109 & 2 & 2 & - \\
\hline 31 & LAM4120 & 3 & _- & _- \\
\hline 32 & LAM4236 & 6 & _ & _ \\
\hline 33 & LAM4241 & 15 & & \\
\hline 34 & LAM4304 & 41 & 10 & 6 \\
\hline 35 & LAM4644 & 11 & 2 & - \\
\hline 36 & LAM4717 & 36 & 8 & - \\
\hline 37 & LAM4767 & 1 & 2 & _ \\
\hline 38 & LAM4783 & 69 & 10 & - \\
\hline 39 & LAM4816 & 102 & 19 & 5 \\
\hline 40 & LAM4852 & 46 & 4 & - \\
\hline 41 & LAM4861 & 5 & - & _ \\
\hline 42 & LAM5237 & 59 & 6 & - \\
\hline 43 & LAM5278 & 29 & 5 & \\
\hline 44 & LAM5279 & 201 & 8 & 5 \\
\hline 45 & LAM5282 & 153 & 7 & - \\
\hline 46 & LAM5318 & 27 & & - \\
\hline 47 & LAM5353 & 206 & 4 & - \\
\hline 48 & LAM5771 & 33 & 3 & _ \\
\hline 49 & LAV0001-13 & 28 & & - \\
\hline 50 & LAV0006-15 & 112 & 12 & 11 \\
\hline 51 & LAV0007-14 & 148 & 12 & 8 \\
\hline 52 & LAV0008-12 & 55 & 8 & 2 \\
\hline 53 & LAV0031-14 & 2 & - & - \\
\hline 54 & LAV0033-14 & 192 & 20 & _- \\
\hline 55 & LAV0061-14 & 2 & 2 & - \\
\hline 56 & NAFTAN & 133 & 125 & 1 \\
\hline & Total & 4577 & 2207 & 140 \\
\hline
\end{tabular}


SDT y viceversa como se presenta en la ecuación 1 y ecuación 2 y los valores de $\mathrm{HCO}_{3}$ - faltantes se calcularon a partir de la alcalinidad como se presenta en la ecuación 3 (Freeze et al, 1979).

Conductividad Electrica $\mu \mathrm{S} / \mathrm{cm}=\frac{\text { Solidos Disueltos Totales } m g l}{0.65}$

Solidos Disueltos Totales $m g l=$ Conductividad Electrica $\frac{\mu \mathrm{S}}{\mathrm{cm}} * 0.65$

Bicarbonatos $\frac{m g}{l}=1.22 *$ Alcalinidad $\frac{m g}{l} \operatorname{CaCo} 3$

Posterior a esto y como procedimiento para asegurar la calidad de las muestras compiladas se calculó el valor de error analítico del balance iónico (Freeze et al., 1979), de manera que la suma de la concentración en miliequivalentes de cationes debe ser igual a la suma de las concentraciones en miliequivalentes de aniones, como se indica en la ecuación 4 .

$$
\% \text { de Error Analítico }=\frac{\sum \text { aniones }-\sum \text { cationes }}{\sum \text { aniones }+\sum \text { cationes }} * 100
$$

Según Hounslow (1995) y Custodio et al. (2001), un error analítico del balance iónico menor o igual al +/- el 10\% se considera aceptable. Con base en los criterios anteriores, de las 2207 muestras analísticas solo 289 cumplían los estándares (análisis de iones mayoritarios completos) para poder calcular el \% de Error analítico; de estos 289 registros, solo 140 cumplieron el porcentaje aceptable del error analítico del balance iónico. La ubicación y tipo de las muestras seleccionadas se presenta en la figura 4.

\subsection{ANÁLISIS ESTADÍSTIGO MULTIVARIADO}

El Análisis Estadístico Multivariado (AEM) es una herramienta que implementada en el análisis fisicoquímico del agua permite la identificación de

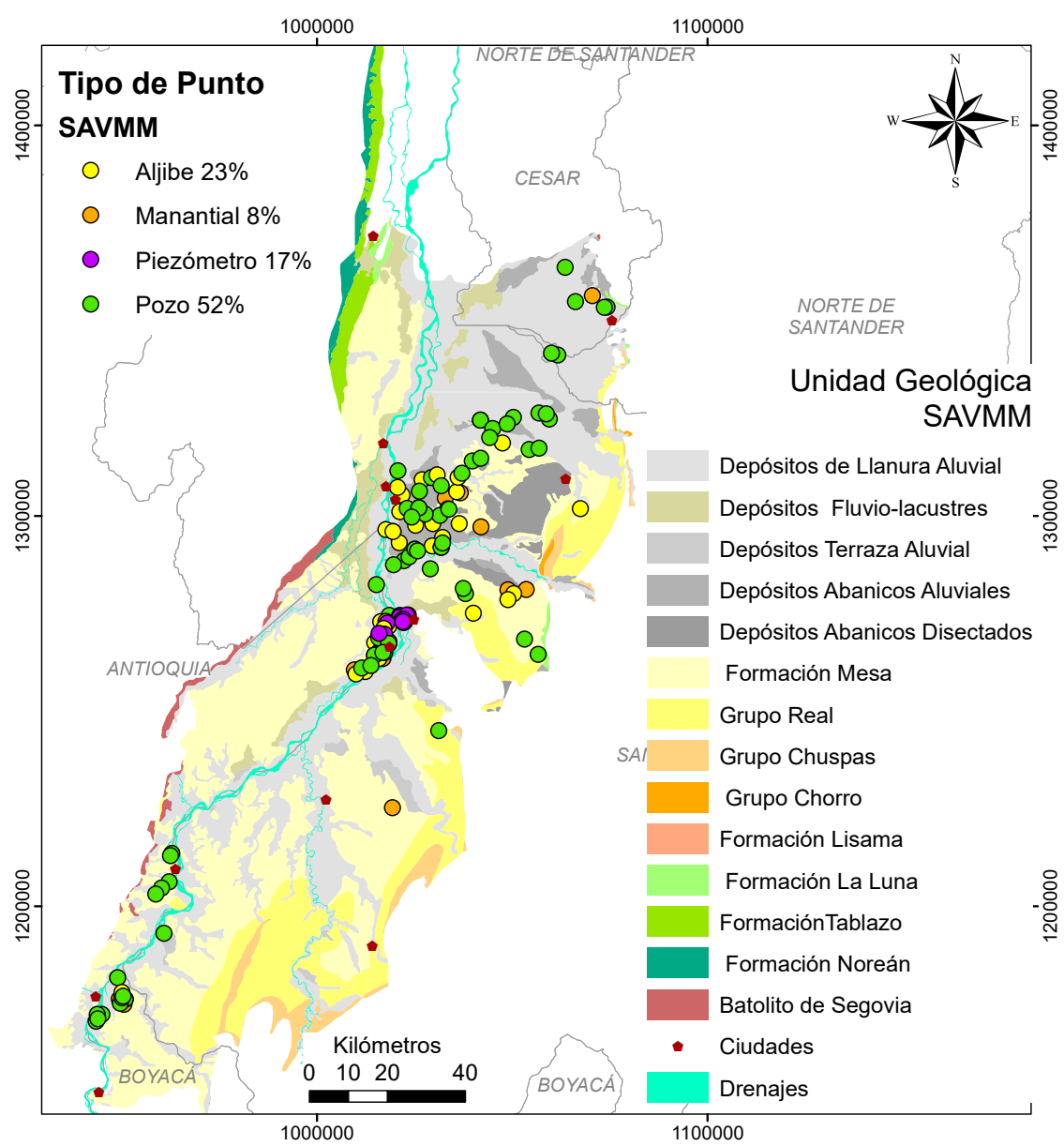

Figura 4 Distribución espacial y tipo de muestra de las 140 muestras que cumplen con los parámetros de calidad para el análisis hidrogeoquímico del SAVMM (Escala 1:980000 - Modificado de IDEAM, 2014). 
grupos distintivos de las muestras, así como las correlaciones entre sus parámetros (Cloutier et al., 2008). El AEM se realizó utilizando el paquete estadístico FactoMineR (Lê et al., 2008) incluido en el programa estadístico $\mathrm{R}$ ( $\mathrm{R}$ Development Core Team, 2011). El conjunto de datos utilizado para el AEM consistió en una base de datos de 140 muestras por 12 parámetros, 10 activos $\left(\mathrm{pH}, \mathrm{Ca}^{2+}\right.$, $\mathrm{Na}^{+}, \mathrm{K}^{+}, \mathrm{Mg}^{2+}$, Fe total, $\mathrm{Cl}^{-}, \mathrm{SO}_{4}{ }^{2-}, \mathrm{HCO}_{3}{ }^{-}$y $\mathrm{NO}_{3}{ }^{-}$) y 2 suplementarios ( $\mathrm{CE}$ y $\mathrm{SDT})$. También se tuvo en cuenta la variable cualitativa tipo de punto (pozo, aljibe, manantial y piezómetro). Los valores de concentración inferiores al límite de detección de los métodos analíticos fueron sustituidos por la mitad del valor del límite de detección (Farnham $e t$ al., 2002). En caso de que la población no tuviera un comportamiento normal, los datos fueron normalizados según el método empleado por Cloutier et al., (2008). En este método de normalización, los parámetros con altos coeficientes de asimetría que no seguían una distribución normal fueron normalizados (James et al., 2016) dado que el método estadístico asume la normalidad de los datos. Los parámetros $\mathrm{pH}, \mathrm{Mg}^{2+}$ y $\mathrm{HCO}_{3}^{-}$se conservaron en su estado original, mientras que se realizó una transformación logarítmica a los parámetros $\mathrm{Ca}^{2+}$, $\mathrm{Na}^{+}, \mathrm{K}^{+}, \mathrm{Fe}$ total, $\mathrm{SO}_{4}{ }^{2}$ y una transformación de raíz cuadrada a los parámetros $\mathrm{Cl}^{-}$y $\mathrm{NO}_{3}^{-}$. Posteriormente, con el fin de eliminar el efecto de las diferentes magnitudes que presenta cada uno de los parámetros, los datos fueron estandarizados (Canavos, 1987), como se describe en la Ecuación 5.

$$
Z i j=\frac{X i j-\mu j}{\sigma j}
$$

Donde Zij es el valor estandarizado del parámetro j para la muestra i, $X i j$ el valor original de la muestra i en el parámetro $j, \mu j$ el valor promedio del parámetro $j$ y oj la desviación estándar del parámetro $j$. Esta operación deja todos los parámetros estandarizados a una distribución que tiene valor promedio 0 y desviación estándar 1 , y se utiliza para asegurar que cada variable tenga el mismo peso en el AEM (Cloutier et al., 2008).
Una vez que se obtuvo el conjunto de datos normalizado y estandarizado, se realizó un AEM utilizando el ACP y el AAJ. El ACP es una técnica estadística utilizada para descifrar patrones dentro de grandes conjuntos de datos (Farnham et al., 2003). Para identificar los patrones químicos que representan las muestras seleccionadas en este estudio, se utilizó una representación geométrica en donde cada muestra se representa como un punto en un sistema de ejes (en este estudio fueron 10 ejes que obedecen al número de parámetros activos). El valor de cada parámetro constituye la coordenada sobre cada eje; sin embargo, los puntos fueron representados en un plano (2 ejes) y aunque su interpretación es abstracta, preserva todas las propiedades de un plano cartesiano tradicional (Lebart et al., 1995). Observar esta representación es el objeto del ACP y esto se logra proyectando el conjunto de muestras sobre nuevos ejes y planos. La solución para todos los ejes se resuelve encontrando los valores propios y sus respectivos vectores propios asociados y ordenándolos de mayor a menor. Como resultado, se obtienen ejes llamados "componentes principales" que resumen la información (Lang, 1990). Los componentes seleccionados para este estudio fueron aquellos cuyo valor propio fuera mayor a uno, de manera que se garantice se explique la mayor parte de la varianza del sistema (Davis, 2002). La interpretación geoquímica de los componentes determinados proporciona una visión de los principales procesos dominantes que pueden controlar la distribución de las variables hidroquímicas (Güler et al., 2002).

Posterior al ACP, se realizó un AAJ, para identificar firmas químicas similares entre las muestras y así aglomerarlas en grupos. Para lograr grupos homogéneos se combinaron dos métodos (Everitt et al., 2011 ): el método de Ward (clasificación jerárquica) y el método de centros móviles usando la distancia euclidiana (clasificación no jerárquica). Primero se realizó una clasificación jerárquica con el método de Ward y se construyó una gráfica en forma de árbol "dendograma" y como número sugerido de grupos; se generó una línea horizontal 
Tabla 2. Estadística descriptiva de las 140 muestras objeto de análisis (las concentraciones de los iones analizados, así como los SDT se expresan en $\mathrm{mg} / \mathrm{L}$, el $\mathrm{pH}$ en sus unidades y la $\mathrm{CE}$ en $\mu \mathrm{S} / \mathrm{cm}$ ).

\begin{tabular}{|c|c|c|c|c|c|c|c|c|c|}
\hline Parámetros & Media & Mediana & Mínimo & Máximo & $\begin{array}{c}\text { Desviación } \\
\text { estándar }\end{array}$ & Varianza & $\begin{array}{c}\text { Coeficiente } \\
\text { de } \\
\text { Variación }\end{array}$ & Asimetría & $\begin{array}{c}\text { Asimetría } \\
\text { Corregida }\end{array}$ \\
\hline $\mathrm{Ca}^{2+}$ & 12.9 & 5.6 & 0.1 & 67.9 & 15.6 & 244.8 & $121 \%$ & 1.7 & 0.3 \\
$\mathrm{Cl}^{-}$ & 10.1 & 3.3 & 0.05 & 123.6 & 18.8 & 354.4 & $186 \%$ & 4.1 & 2.3 \\
$\mathrm{C.E}$. & 253.3 & 186.2 & 10 & 1.120 & 230.5 & 53.136 & $91 \%$ & 1.7 & 0.5 \\
$\mathrm{Fe}$ total & 7.1 & 0.6 & 0.05 & 151 & 20.9 & 435.9 & $294 \%$ & 5.2 & -2.8 \\
$\mathrm{HCO}_{3}^{-}$ & 74.5 & 39.2 & 1.2 & 350 & 92.3 & 8.517 & $124 \%$ & 1.5 & 1.4 \\
$\mathrm{~K}^{+}$ & 2.7 & 1.9 & 0.05 & 23 & 3.2 & 10.2 & $119 \%$ & 3.6 & -1 \\
$\mathrm{Mg}^{2+}$ & 4.0 & 1.8 & 0.05 & 22.1 & 4.7 & 22.1 & $118 \%$ & 1.6 & -1.5 \\
$\mathrm{Na}^{+}$ & 15.4 & 7.9 & 0.3 & 216 & 28.3 & 803.6 & $184 \%$ & 5.0 & 0.2 \\
$\mathrm{NO}_{3}^{-}$ & 0.7 & 0.3 & 0.05 & 15.3 & 1.6 & 2.6 & $229 \%$ & 6.2 & 2.1 \\
\hline $\mathrm{pH}$ & 6.8 & 6.8 & 4.4 & 8.6 & 0.7 & 0.5 & $10 \%$ & 0 & 0 \\
$\mathrm{SDT}^{-}$ & 144.7 & 111.5 & 6.5 & 728 & 130.2 & 1.6958 & $90 \%$ & 1.5 & 0.5 \\
$\mathrm{SO}_{4}^{2-}$ & 4.5 & 2.5 & 0.1 & 57.6 & 7.4 & 55.2 & $164 \%$ & 4.6 & -1 \\
\hline
\end{tabular}

"línea fenotípica" que cortó el dendograma en el número de grupos que se determinó como óptimo local. Con este número de grupos entonces el método no jerárquico de centros móviles asignó de manera óptima las muestras a cada centro de gravedad y la iteración hasta llegar a un óptimo absoluto.

\subsection{ANÁLISIS GRÁFICOS}

La exploración y análisis de la información hidroquímica del agua subterránea se realizó mediante métodos gráficos teniendo en cuenta el contenido de los macro-componentes (Güler et al., 2002). Para este estudio se utilizaron los diagramas de Piper y Stiff usando el programa Diagrammes (Simler, 2013). El diagrama Piper (1944), es una de las técnicas de visualización más utilizadas para la exploración gráfica de los datos químicos del agua subterránea. La combinación de un diagrama ternario de la composición catiónica principal $\left(\mathrm{Ca}^{2+}\right.$, $\mathrm{Na}^{+}, \mathrm{K}^{+}$y $\mathrm{Mg}^{2+}$ y un diagrama ternario de la composición aniónica principal $\left(\mathrm{Cl}^{-}, \mathrm{SO}_{4}^{2-}, \mathrm{HCO}_{3}^{-}\right.$ y $\mathrm{NO}_{3}^{-}$) en un diamante central, lo convierten en una herramienta invaluable para identificar grupos o facies hidroquímicas e interpretar algunos procesos químicos que representa el conjunto de datos por analizar (Peeters, 2014). Por otro lado, en el diagrama de $\mathrm{Stiff}$ (1951) el contenido de iones es presentado por un polígono cuyo lado izquierdo describe el contenido de cationes mayores y el lado derecho, los aniones mayores. La distancia desde el eje central es proporcional a las concentraciones en $(\mathrm{meq} / \mathrm{L})$ de iones individuales. La ventaja de este patrón es que el agua de composición química similar tiene geometría y forma semejantes (Tikhomirov, 2016).

\section{Resultados y discusión}

Se identificaron los posibles procesos geoquímicos que controlan la calidad y composición química del agua subterránea del SAVMM. Por medio del ACP se identificaron 3 componentes que explican el 59,4\% de la varianza del sistema y a través del AAJ se definieron 6 grupos que representan la información química de las 140 muestras utilizadas para este estudio. A continuación se describe los resultados obtenidos.

\subsection{QUÍMICA DEL AGUA DEL SAVMM}

Con base en las concentraciones medias de los parámetros analizados Tabla 2 se infiere que la composición química del agua subterránea del ASVAMM está dominada por los cationes $\mathrm{Ca}^{2+}$, $\mathrm{Na}^{+}$y los aniones $\mathrm{HCO}_{3}^{-}$, y en una menor pro- 
porción por $\mathrm{Cl}^{-}$. Los valores máximos y mínimos de los cationes y aniones reflejan el grado de heterogeneidad química del agua subterránea como resultado de los diferentes procesos geoquímicos presentes en el área de estudio.

Con base en el diagrama de Piper (Figura 5) se determinó que la facie hidrogeoquímica bicarbonatada cálcica es el tipo de agua dominante en el área de estudio (51.4\%), seguida de aguas bicarbonatada sódica $(26.4 \%)$ y en menor proporción agua clorurada sódica $(15 \%)$ y magnésica $(7.1 \%)$. Las muestras del tipo de agua bicarbonatada cálcica en su mayoría están ubicadas en los depósitos Cuaternarios, agua subterránea poco profunda con tiempos cortos de residencia, se puede ver su distribución espacial en la Figura 8, correspondiente al grupo 1. Se infiere, por su poca concentración de iones disueltos, que este tipo de agua representa agua de infiltración reciente y su firma química no es muy diferente del agua de lluvia. El tipo de agua bicarbonatada sódica es también importante en la zona, ubicada en su mayoría en los depósitos de llanura aluvial del Río Magdalena (Cañas et al., 2019), en los cuales, se presentan pro- cesos de alteración de silicatos. Los tipos de agua restantes son tipos de agua intermedios entre los mencionados anteriormente (Figura 5).

\subsection{ANÁLISIS DE GOMPONENTES PRINGIPALES - ACP}

Para este estudio se tomaron los tres primeros componentes principales con valores propios mayores a 1 (Davis, 2002), los cuales explican el $59.4 \%$ de la varianza total en el conjunto de datos (Tabla 3). Aunque la varianza acumulada para los tres componentes seleccionados no es alta, los resultados obtenidos pueden explicar con éxito las interrelaciones entre las variables. El componente principal 1 explica la mayor cantidad de la varianza con un $35.4 \%$ (Tabla 3 ) y se caracteriza por pesos positivos altos de los cationes $\mathrm{Ca}^{2+}, \mathrm{Na}^{+}, \mathrm{K}^{+}, \mathrm{Mg}^{2+}$ y el anión $\mathrm{HCO}_{3}^{-}$. Los parámetros presentes pueden indicar procesos de alteración de silicatos presentes en la zona, dado que los iones representados por este componente son débilmente retenidos por la estructura silicatada, por lo cual, quedan fácilmente en solución. El componente principal 2 explica el $12 \%$ de la varianza total (Tabla 3) y

\section{Diagrama de Piper SAVMM}

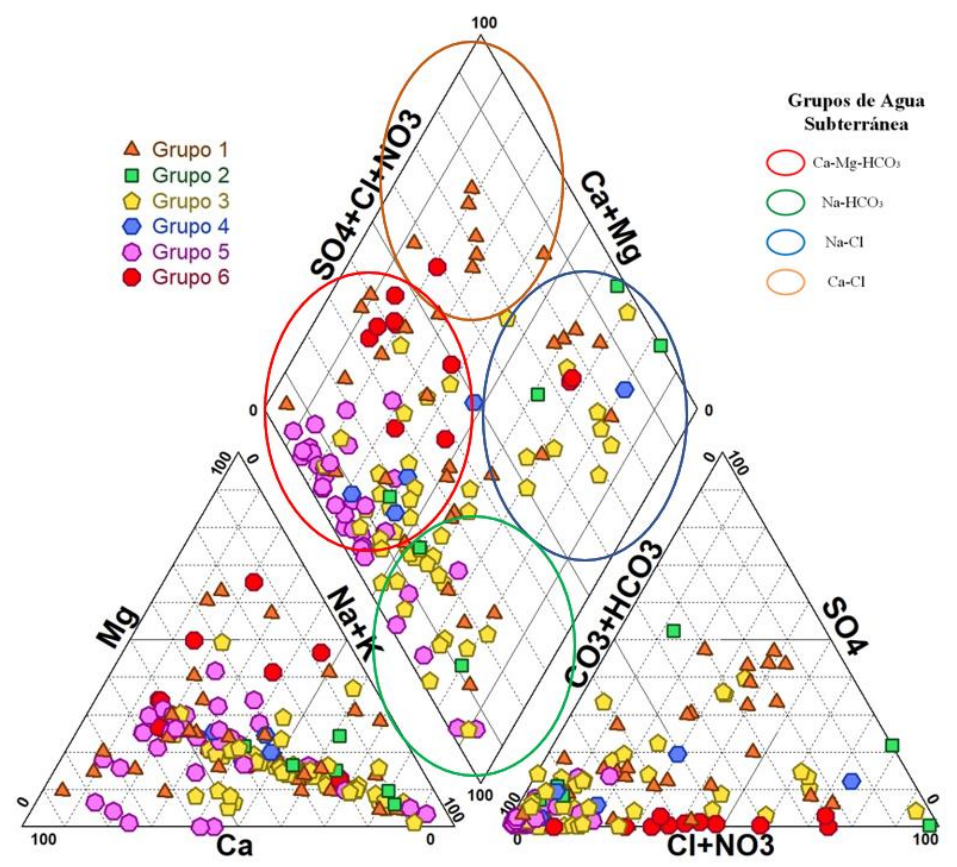

Figura 5 Diagrama de Piper que muestra los 6 grupos establecidos a través del Análisis de Agrupamiento Jerárquico para las 140 muestras. 
Tabla 3. Resultados del Análisis de Componentes Principales y varianza explicada. En negrilla se resaltan los pesos de las variables (parámetros) mejor representados por cada componente principal. Los valores cercanos a 1 indican que el parámetro está mejor representado en el componente principal.

\begin{tabular}{cccc}
\multicolumn{1}{l}{ Parámetro } & Componente 1 & Componente 2 & Componente 3 \\
\hline $\mathrm{pH}$ & 0.438 & -0.251 & -0.464 \\
$\mathrm{Ca}^{2+}$ & $\mathbf{0 . 8 7 5}$ & -0.041 & -0.143 \\
$\mathrm{Na}^{+}$ & $\mathbf{0 . 7 5 8}$ & 0.251 & -0.145 \\
$\mathrm{~K}^{+}$ & 0.694 & 0.155 & -0.057 \\
$\mathrm{Mg}^{2+}$ & $\mathbf{0 . 7 7 0}$ & -0.011 & 0.135 \\
$\mathrm{Fe}$ total & 0.216 & -0.546 & 0.527 \\
$\mathrm{Cl}^{-}$ & 0.499 & 0.078 & $\mathbf{0 . 6 0 6}$ \\
$\mathrm{SO}^{2-}$ & 0.162 & $\mathbf{0 . 7 0 3}$ & -0.109 \\
$\mathrm{HCO}_{3}{ }^{-}$ & $\mathbf{0 . 7 8 2}$ & -0.252 & -0.062 \\
$\mathrm{NO}_{3}{ }^{-}$ & 0.045 & 0.442 & 0.512 \\
Valor Propio & 3.542 & 1.210 & 1.200 \\
Varianza (\%) & 35.420 & 12.097 & 12.002 \\
Var. Acumulada & 35.420 & 47.517 & 59.519
\end{tabular}

está caracterizado por valores negativos, con altos pesos del $\mathrm{SO}_{4}^{2-}$. Este componente está asociado a actividades antrópicas presentes en la zona de estudio (centros poblados y agroindustria).

Por último, el componente principal 3 explica el $12 \%$ de la varianza total, y presenta altos pesos de $\mathrm{Cl}^{-}$, probablemente de origen antropogénico, condición que parece estar asociada a una inadecuada gestión del agua de producción (aguas con elevadas concentraciones de cloruro) derivada de la extracción de petróleo presente en el área de estudio, esta hipótesis se desarrolla con más detalle más adelante. En el plano factorial conformado por los componentes 1 y 2 (Figura 6a) todas las correlaciones son positivas dado que ninguna flecha (parámetro) está en dirección opuesta de otra. El primer eje (dimensión 1) recoge la mayoría de la inercia - dispersión de los puntos en el plano y separa las muestras de izquierda a derecha. Por lo tanto, en el lado izquierdo del plano factorial (proyección de las muestras) están ubicadas las muestras de agua con menor concentración de iones y al lado derecho las muestras con mayores concentraciones de iones. Por esta condición, se considera que el componente principal 1 representa los procesos de interacción agua roca que dan origen a la mineralización del agua. Las muestras menos mineralizadas se encuentran ubicadas en zonas de recarga del sistema acuífero y las más mineralizadas al interior de la cuenca y a mayores profundidades con mayor tiempo de residencia en el sistema hidrogeológico. Esta hipótesis es apoyada con la proyección de la variable cualitativa "tipo de punto" en el plano factorial (Figura 6b). Los manantiales están ubicados al lado izquierdo donde el agua es menos mineralizada (concentraciones de conductividad eléctrica promedio de $77 \mu \mathrm{S} / \mathrm{cm}$ ) y los pozos y piezómetros corresponden al agua más mineralizada y están ubicados al lado derecho (concentraciones de conductividad eléctrica promedio de $513.2 \mu \mathrm{S} / \mathrm{cm}$ ). El segundo eje (componente 2) separa las muestras de arriba abajo. Las muestras representadas arriba del plano factorial están caracterizadas por tener altas concentraciones de los aniones $\mathrm{SO}_{4}{ }^{2-} \mathrm{y}$ $\mathrm{NO}_{3}{ }^{-}$(Figura 6c). La presencia de estos aniones en el área de estudio puede ser explicada por la actividad agroindustrial que utiliza fertilización química, la cual contiene $\mathrm{SO}_{4}{ }^{2-}$ y $\mathrm{NO}_{3}{ }^{-}$y estos a su vez, puede infiltrase y llegar al agua subterránea.

Las muestras que se encuentran debajo del plano factorial presentan altas concentraciones de Fe total y bajas concentraciones $\mathrm{SO}_{4}{ }^{2-}$ y $\mathrm{NO}_{3}$. Es posible que debido al ambiente reductor que es característico de los sistemas hidrogeológicos, dado la baja concentración de oxigeno disuelto, se presente un proceso de reducción de sulfatos y nitratos que por lo general conlleva a un aumento de las concentraciones de hierro (Custodio et al., 2001). Por lo cual, el componente principal 2, probablemente representa los procesos redox, situación que debe ser estudiada con mayor detalle determinando valores de potencial oxidorreducción en el agua subterránea presente en el área de estudio.

\subsection{ANÁLISIS DE AGRUPAMIENTO JERÁRQUICO (AAJ)}

La clasificación de las muestras utilizadas en grupos se basa en una observación visual del dendograma (Güler et al., 2002). La posición de la línea fenotípica que cruza el dendograma permitió la conformación de 6 grupos que resumen la información química de las 140 muestras (Figura 7a). Los valores promedio para cada parámetro revelan 
alguna tendencia entre los grupos identificados. A partir de los valores medios de las concentraciones de cada grupo se realizó un diagrama de Stiff (Figura 7b) que permitió identificar diferencias y similitudes entre cada grupo. En la tabla 4 se resume la conformación de los 6 grupos.

Para los grupos 2, 3 y 4 el diagrama de Stiff presenta formas similares que se van ensanchando del grupo 2 al 4 con los iones $\mathrm{Na}^{+}$y $\mathrm{HCO}_{3}{ }^{-}$como dominantes. Por otra parte, el grupo 1 lo conforman las muestras de agua menos mineralizada y

a)

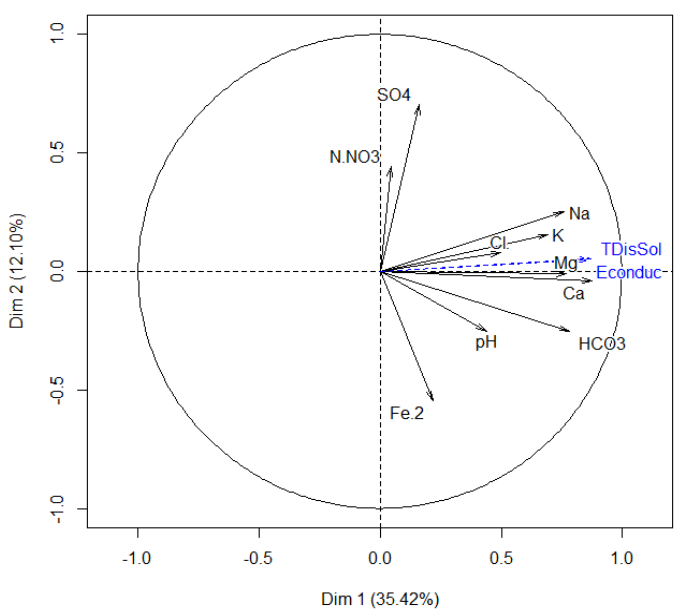

su forma corresponde a la mayor concentración de los iones $\mathrm{Ca}^{2+}$ y $\mathrm{HCO}_{3}^{-}$(concentraciones de conductividad eléctrica promedio de $77 \mu \mathrm{S} / \mathrm{cm})$. Los grupos 5 y 6 lo conforman las muestras de agua más mineralizada, se observa que en el grupo 5 los iones dominantes son $\mathrm{Na}^{+}, \mathrm{Ca}^{2+}$ y $\mathrm{HCO}_{3}^{-}$, y en el grupo 6 los iones dominantes son $\mathrm{el} \mathrm{Ca}^{2+}, \mathrm{HCO}_{3}^{-} \mathrm{y}$ el $\mathrm{Cl}^{-}$(concentraciones de conductividad eléctrica promedio de $513.2 \mu \mathrm{S} / \mathrm{cm})$.

Los resultados del AAJ son consistentes con los resultados del ACP. Las muestras del grupo 1 están

b)

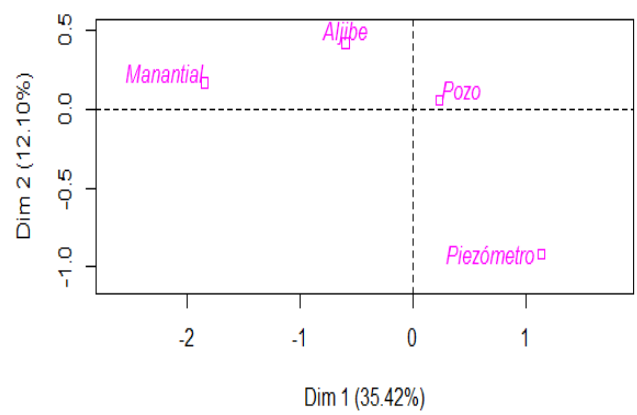

c)

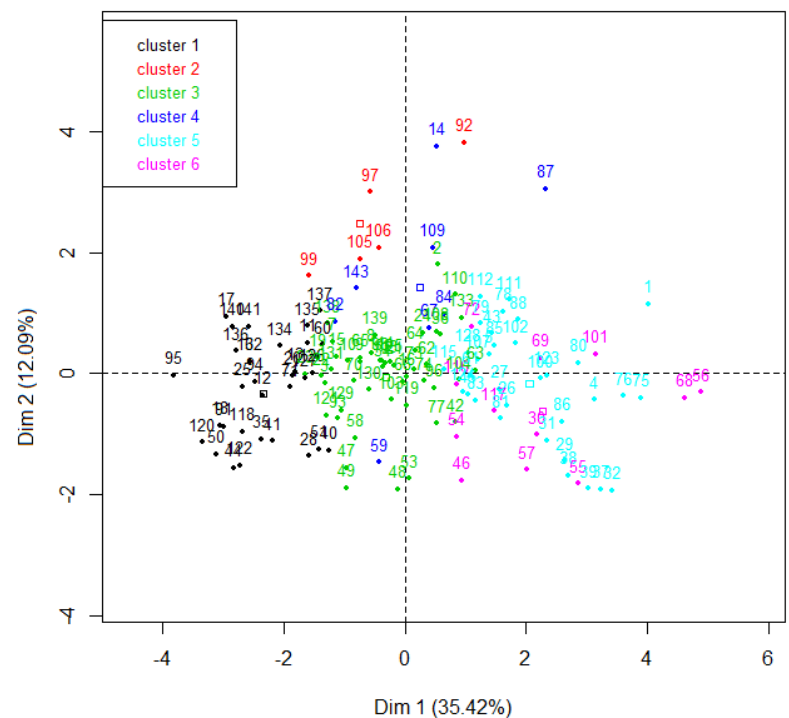

Figura 6 (a) Trazado de los parámetros en el círculo de correlaciones respecto a los componentes principales (dimensiones) 1 y 2. (b) Variable cualitativa proyectada en el plano factorial respecto a los componentes principales (dimensiones) 1 y 2 (c) proyección de las 140 muestras de agua subterránea según resultados del Análisis de Agrupamiento Jerárquico en el plano factorial respecto a los componentes principales (dimensiones) 1 y 2. 
Tabla 4. Características químicas promedio de cada grupo identificado con el AAJ (las concentraciones de los iones analizados, así como los SDT se expresan en $\mathrm{mg} / \mathrm{L}$, el $\mathrm{pH}$ en sus unidades y la $\mathrm{CE}$ en $\mu \mathrm{S} / \mathrm{cm}$ ).

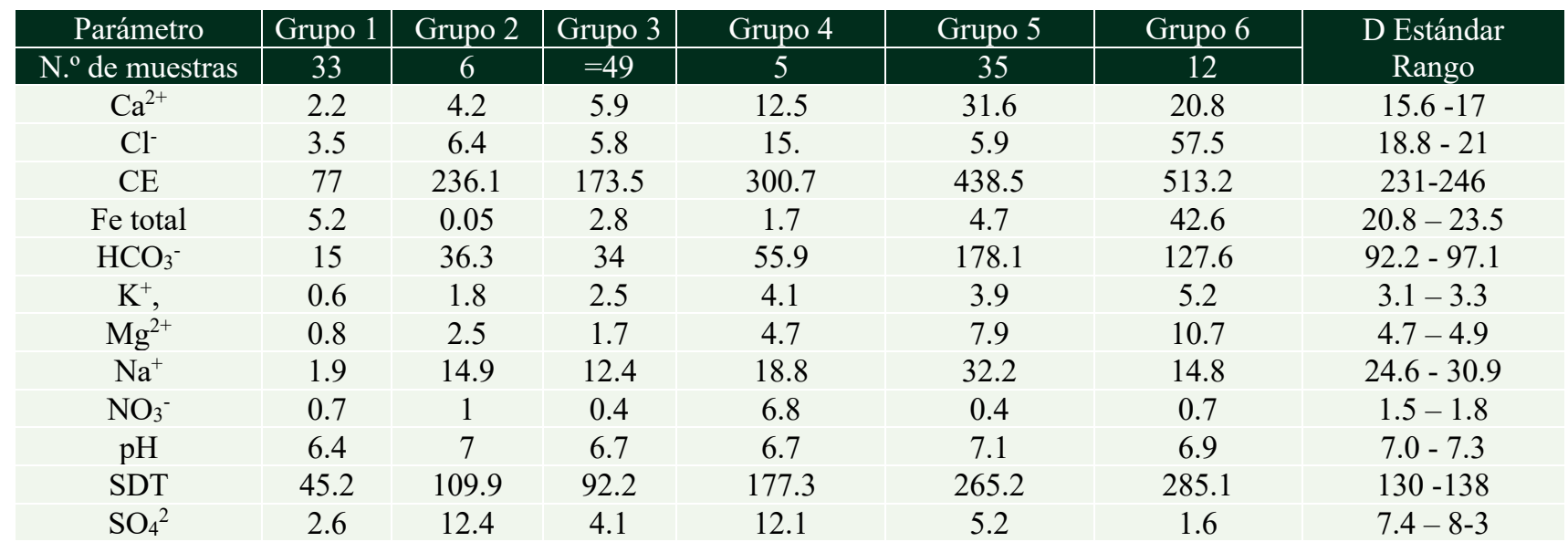

situadas en el lado medio-izquierdo del plano factorial y corresponden a las muestras menos mineralizadas, en su mayoría manantiales (Figura 6c) mientras que las muestras de los grupos 5 y 6 están situadas en el lado derecho y corresponden a las muestras más mineralizadas, en su mayoría conformadas por pozos y piezómetros. Las muestras de los grupos 2, 3 y 4 se encuentran en el centro del plano factorial y son las muestras con nivel intermedio de mineralización. En consecuencia, la distribución de las muestras en el plano factorial y de acuerdo con el círculo de correlaciones es de las menos mineralizadas: grupo 1 (lado izquierdo sentido contrario a las flechas del círculo de correlaciones); a las más mineralizada: grupo 6 (lado derecho y en dirección a las flechas del círculo de correlaciones), como se presenta en la figura 6a.

A través del diagrama de Mifflin figura 7, se puede evidenciar la situación descrita, donde las muestras del grupo 1 menos mineralizadas están ubicadas en la parte inferior izquierda indicando flujos locales y conforme el agua va volviéndose más mineralizada grupos 5 y 6 , estas se ubican en el superior derecho indicando flujos intermedios. Dada la distribución geográfica de los datos existentes en el SAVMM, y los resultados obtenidos mediante la congregación de los grupos, no es posible determinar una evolución geoquímica del agua subterránea en función de una línea de flujo (Figura 10). Sin embargo, se identificó que el comportamiento de los grupos en función de la mineralización es explicado en gran medida por las profundidades en las cuales fueron tomadas las muestras. Esta tendencia identificada es validada a través del diagrama de dispersión presentado en la figura 8. En este se evidencia que el aumento de la conductividad eléctrica está relacionado con el aumento de la profundidad. El grupo 1 está conformado por muestras someras con tiempos cortos de residencia como manantiales y muestras ubicadas en el extremo izquierdo inferior de la gráfica. Los grupos 2, 3 y 4 tienen valores intermedios de $\mathrm{CE}$ y profundidades intermedias, mientras que las muestras que conforman el grupo 5 en su mayoría fueron tomadas en pozos profundos. Para el grupo 6 se evidencia una anomalía dado que está conformado en su mayoría por piezómetros los cuales son puntos poco profundos, pero son los más mineralizados, lo cual apoya la hipótesis de contaminación por Cl- antropogénico aportado por la industria petrolera que se desarrolla a lo largo del área de estudio, situación que se abarca con mayor detalle más adelante.

\subsection{INTERPRETACIÓN GEOQUÍMICA DE LOS GRUPOS}

Se realizó un análisis de cada grupo en función de las características geológicas e hidrogeológicas del área de estudio. En el análisis hidroquímico 


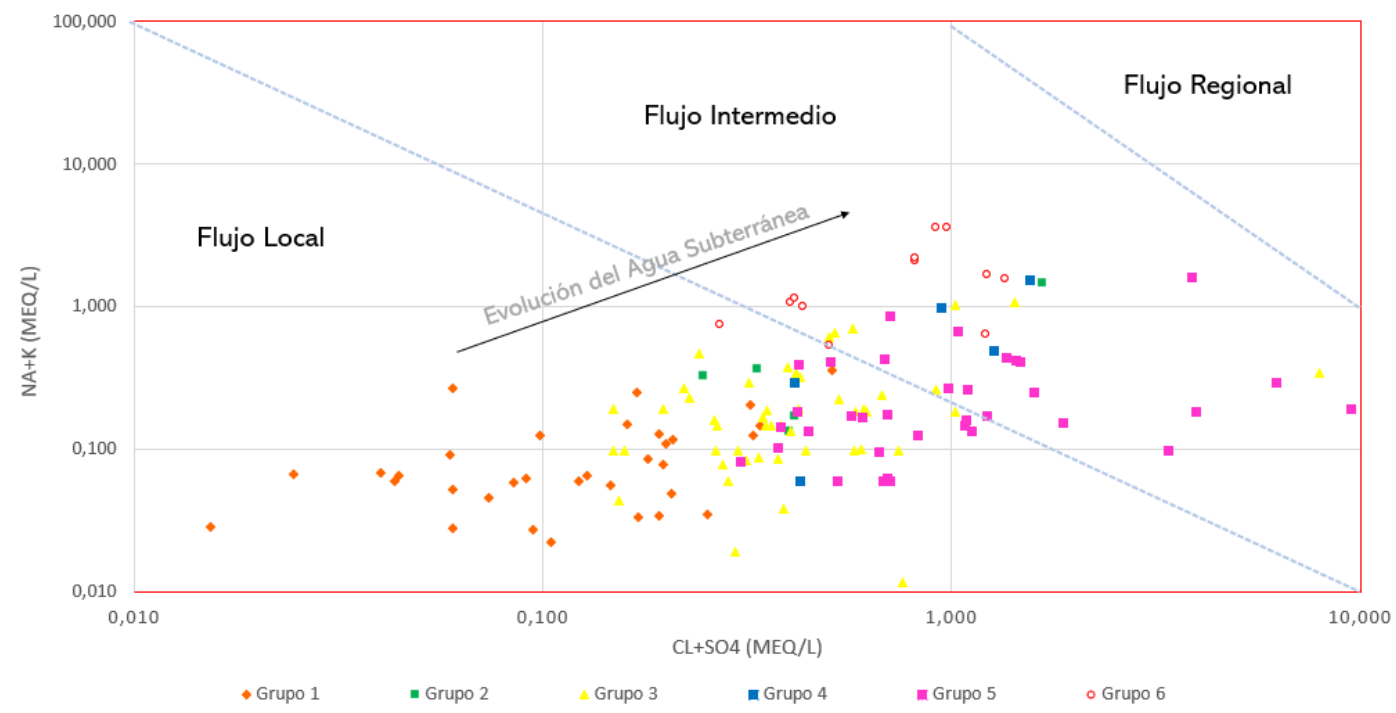

Figura 7 Diagrama de Mifflin, en el que se puede evidenciar que las muestras del grupo 1 están ubicadas en la parte inferior izquierda indicando flujos locales y conforme el agua va volviéndose más mineralizada grupos 5 y 6 , estas se ubican en el extremo superior derecho indicando flujos intermedios.

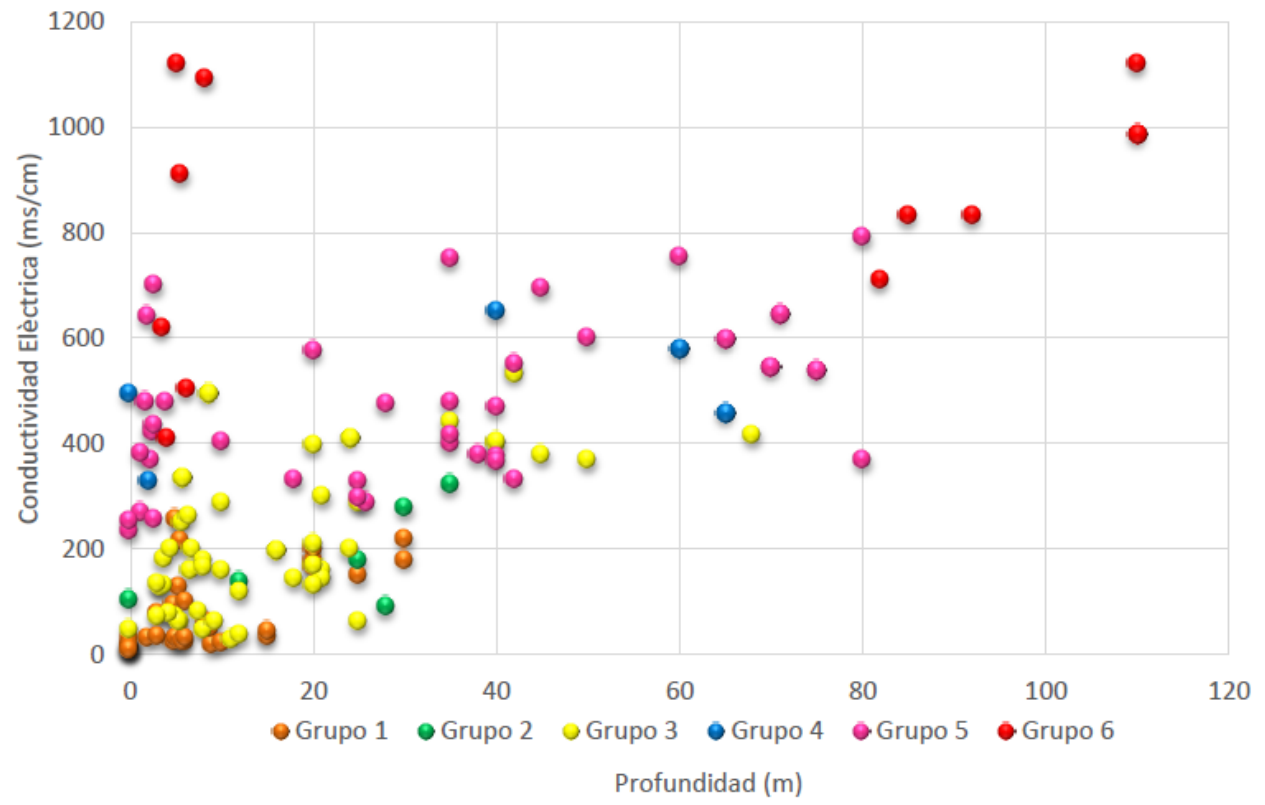

Figura 8 Diagrama de dispersión que muestra el comportamiento de la mineralización de los grupos generados a través del AAJ en función de la profundidad. En este se puede identificar que las muestras del grupo 1 (menos mineralizadas y ubicadas a profundidades menores) están ubicadas al lado izquierdo de la gráfica, caso contrario las muestras de los grupos 5 y 6 (que obedecen a muestras más mineralizadas y ubicadas a mayores profundidades). En caso de las muestras ubicadas a menores profundidades, pero altamente mineralizadas grupo 6, representan las muestras tomadas de los piezómetros y apoyan la hipótesis de contaminación antrópica por Cl-. 
a)

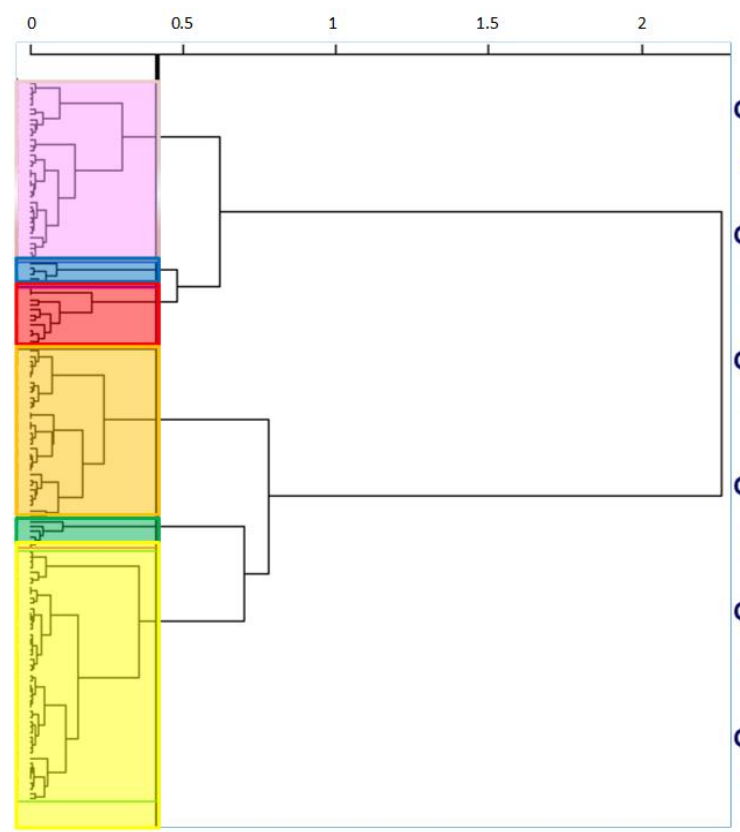

b)

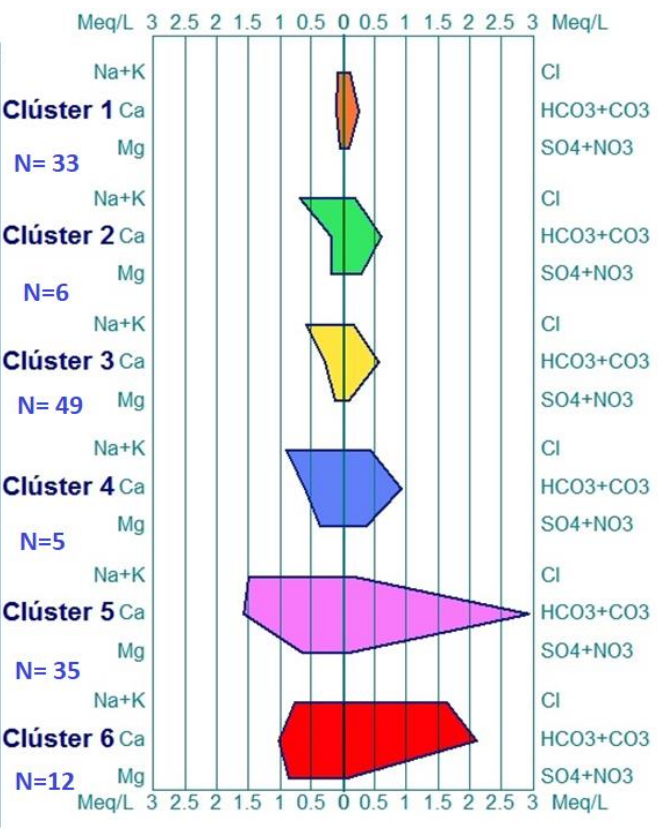

Figura 9 Resultados del Análisis de Agrupamiento Jerárquico: a) Identificación de los grupos en el Dendograma utilizando Ward y Centros Móviles. b) Diagrama de Stiff que muestra la composición química media de los iones mayoritarios de cada uno de los 6 grupos derivados del AAJ.

y en el ACP se logró identificar que los procesos principales que definen la composición química corresponden: Recarga - infiltración de agua meteórica, Disolución - interacción del agua con silicatos y carbonatos y Aporte Antropogénico (contaminación por el desarrollo de actividades agroindustriales realizadas en el área de estudio). $\mathrm{El}$ análisis de los grupos presenta consistencia con los procesos geoquímicos descritos.

El grupo 1 representa el $27 \%$ de las muestras y se caracteriza por tener la concentración más baja de los iones mayoritarios (concentraciones de conductividad eléctrica promedio de $77 \mu \mathrm{S} / \mathrm{cm}$ ), por lo cual son aguas que están ligeramente mineralizadas con tiempos cortos de residencia y flujos locales (Figura 7). La composición química puede deberse a agua que está relacionada con la composición del agua meteórica que interactúa con los niveles acuíferos a través de la recarga natural (Ghesquière et al., 2015). Para los grupos 2, 3 y 4 el diagrama de Stiff presenta formas similares con tendencia al ensanchamiento respecto a los iones dominantes $\mathrm{Na}^{+}$y $\mathrm{HCO}_{3}^{-}$. Estos grupos están ubicados en unidades hidrogeológicas similares; depósitos cuaternarios que componen el SAVMM (Figura 4c), cuyos iones dominantes están asociados

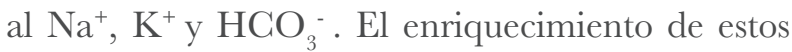
iones puede ser debido a la alteración incongruente de la albita plagioclasa sódica (Freeze et al., 1979) presente en los sedimentos cuaternarios del área de estudio.

En los grupos 2, 3 y 4 también se evidencia un aumento en la concentración de $\mathrm{K}^{+}$que puede ser debido a la disolución de feldespatos potásicos presentes en el área de estudio (Cañas et al., 2019), como las ortoclasas, las cuales están presentes en los fragmentos líticos de la matriz de los cuerpos cuaternarios y neógenos existentes en el SAVMM (Morales et al., 1958), reacción que al igual que la albita es una alteración que produce caolinita (Ecuación 6). De igual forma el enriquecimiento del agua en $\mathrm{Mg}^{2+}$ puede estar asociado a la disolución de dolomita (Ecuación 8). Otra fuente puede ser la disolución de filosilicatos que contienen magnesio en su estructura, lo cuales están presentes en las rocas graníticas que circundan el SAVMM. 


$$
\begin{aligned}
& 2 \mathrm{KAlSi}_{3} \mathrm{O}_{8}(\mathrm{~g})+9 \mathrm{H}_{2} \mathrm{O}(\mathrm{l})+2 \mathrm{H}^{+} \\
& \leftrightarrow \mathrm{Al}_{2} \mathrm{Si}_{2} \mathrm{O}_{5}(\mathrm{OH})_{4}+4 \mathrm{Si}(\mathrm{OH})_{4}+2 \mathrm{~K}^{+} 5
\end{aligned}
$$

Por otra parte, 5 de las 35 muestras que conforman el grupo 5 presentan altas concentraciones de $\mathrm{Na}^{+}$(Figura 9a), lo que hace que la geometría del diagrama de Stiff para este grupo sea atípica. En general, las muestras de este grupo tienen un enriquecimiento importante del catión $\mathrm{Ca}^{2+}$ y se mantiene la tendencia del $\mathrm{HCO}_{3}{ }^{-}$como anión predominante. En su mayoría este grupo está conformado por pozos que captan agua de estratos más profundos (entre 50 y $80 \mathrm{~m}$ aproximadamente) con una mayor evolución geoquímica, la cual explica su mayor mineralización. El principal proceso es la disolución de carbonatos (Cañas et al., 2019), específicamente de los minerales calcita y dolomita que aportan $\mathrm{Ca}^{2+}$ y $\mathrm{HCO}_{3}^{-}$. Estos minerales son solubles al agua, liberando así los iones predominantes en este grupo. En las ecuaciones 7 y 8 (Cañas et al., 2019) se presenta la reacción que representa la disolución de la dolomita y de la calcita, y se evidencia cómo en el proceso se libera el $\mathrm{Ca}^{2+}$ y $\mathrm{HCO}_{3}^{-}$y también el $\mathrm{Mg}^{2+}$, en el agua.

$$
\begin{aligned}
& \mathrm{CO} 2(g)+\mathrm{H} 2 \mathrm{O}(\mathrm{l})+\mathrm{CaCO}_{3}(s) \\
& \leftrightarrow \mathrm{Ca}^{2+}(a c)+2 \mathrm{HCO}_{3}^{-}(a c)
\end{aligned}
$$

$2 \mathrm{CO} 2(g)+2 \mathrm{H}_{2} \mathrm{O}(\mathrm{l})+\mathrm{MgCa}\left(\mathrm{CO}_{3}\right)_{2}(s)$

$\leftrightarrow \mathrm{Ca}^{2+}(a c)+\mathrm{Mg}^{2+}(a c)+4 \mathrm{HCO}_{3}{ }^{-}(a c)$

El proceso de disolución descrito se presenta en la figura 12a y 12b, a través del programa PHREEQG (USGS, 2018) se estimaron los índices de saturación para la dolomita y la calcita con base en la composición química de las muestras. Con el análisis se puede inferir que la disolución es el proceso predominante y no la precipitación, a su vez se identifica que hay un mayor poder de disolución en los grupos 1,2 y 3 que a la vez son los menos mineralizados y que las muestras más mineralizadas como las que están en el grupo 5 ya tienen menor capacidad de disolución debido a su mayor grado de saturación. Otra fuente probable de $\mathrm{Ca}^{2+}$ en el agua puede ser la alteración de piroxenos o anfíboles, presentes en los cuerpos ígneos que afloran en el área de estudio (Cañas et al., 2019).

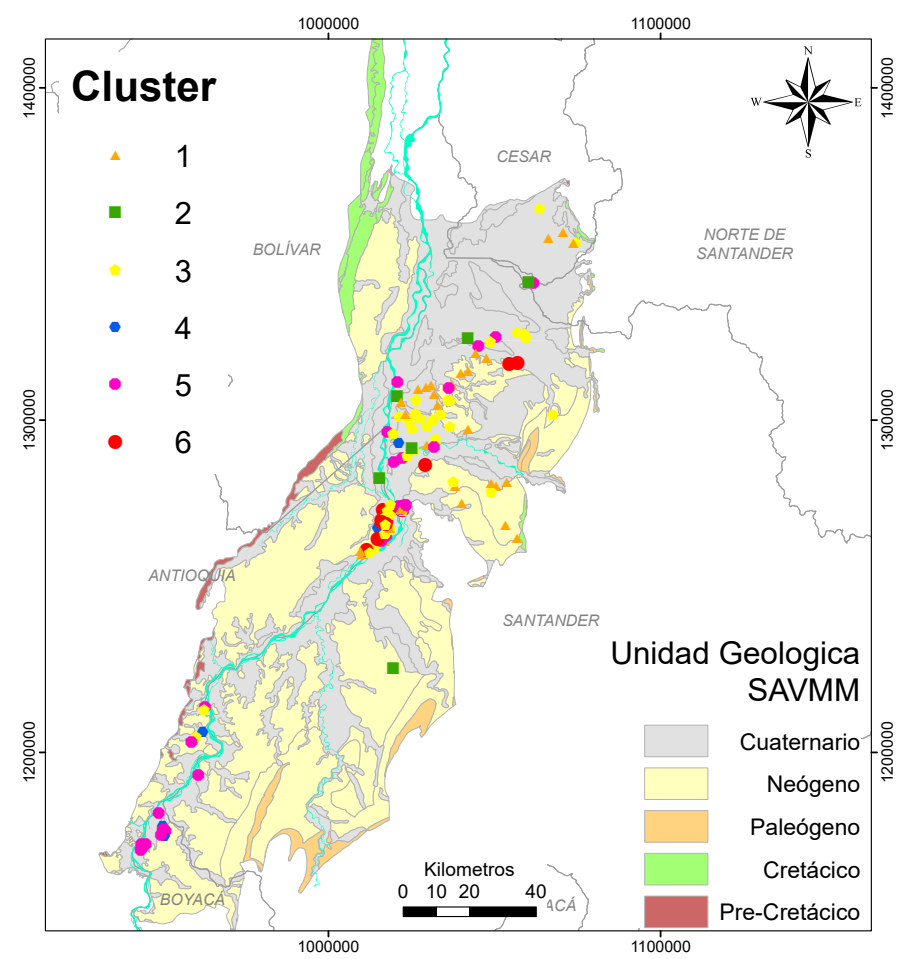

Figura 10 Distribución espacial de las muestras de acuerdo con los resultados del Análisis de Agrupamiento Jerárquico (Escala 1:980000 - Modificado de IDEAM 2014). 
El grupo 6 presenta concentraciones de SDT altos dado que lo conforman las muestras más ionizadas, caracterizados por altas concentraciones de los iones $\mathrm{Ca}^{2+}$ y Cl-. El diagrama de Stiff para este grupo, presenta una geometría irregular que sugiere una alteración externa al sistema hidrogeológico del área de estudio. A diferencia de los demás grupos donde el grado de mineralización es en cierta medida función de la profundidad (a mayor profundidad más mineralizados), este grupo es representado en su mayoría por piezómetros, lo cuales son instalados en zonas que requieren un control regular del agua subterránea poco profunda. Para este caso particular los piezómetros fueron instalados para el monitoreo del agua subterránea en áreas licenciadas para la producción de hidrocarburos y en su mayoría están ubicados en el área de influencia del campo de producción petrolera Casabe (8 de las 12 muestras consideradas), como se observa en la figura $13 \mathrm{~b}$.
En este contexto cabe resaltar que en la extracción de hidrocarburos se generan "aguas de producción", las cuales se caracterizan por su alto grado de salinidad, específicamente por tener altas concentraciones de $\mathrm{Cl}^{-}$. El inadecuado manejo de esta agua podría ser uno de los principales causantes de la contaminación de acuíferos, ya sea por derrames en superficie, fallas en el revestimiento de las piscinas de almacenamiento o por falta de integridad de los pozos inyectores destinados para la disposición final de estas aguas (USEPA, 2016). En el Campo Casabe se generan cerca de 163.000 barriles de agua de producción por día (ANLA, 2015), unos 26 millones de litros de agua, los cuales deben ser gestionadas según lo considerado en la licencia ambiental. En la figura 11 se identifican las muestras de este grupo debido a una alta relación de $\mathrm{Cl}^{-} /\left(\mathrm{Cl}^{-}+\mathrm{HCO}_{3}\right)$. Estas observaciones indican que el aporte de $\mathrm{Cl}^{-}$en el agua subterránea es de origen antropogénico (Montcoudiol, et al.,

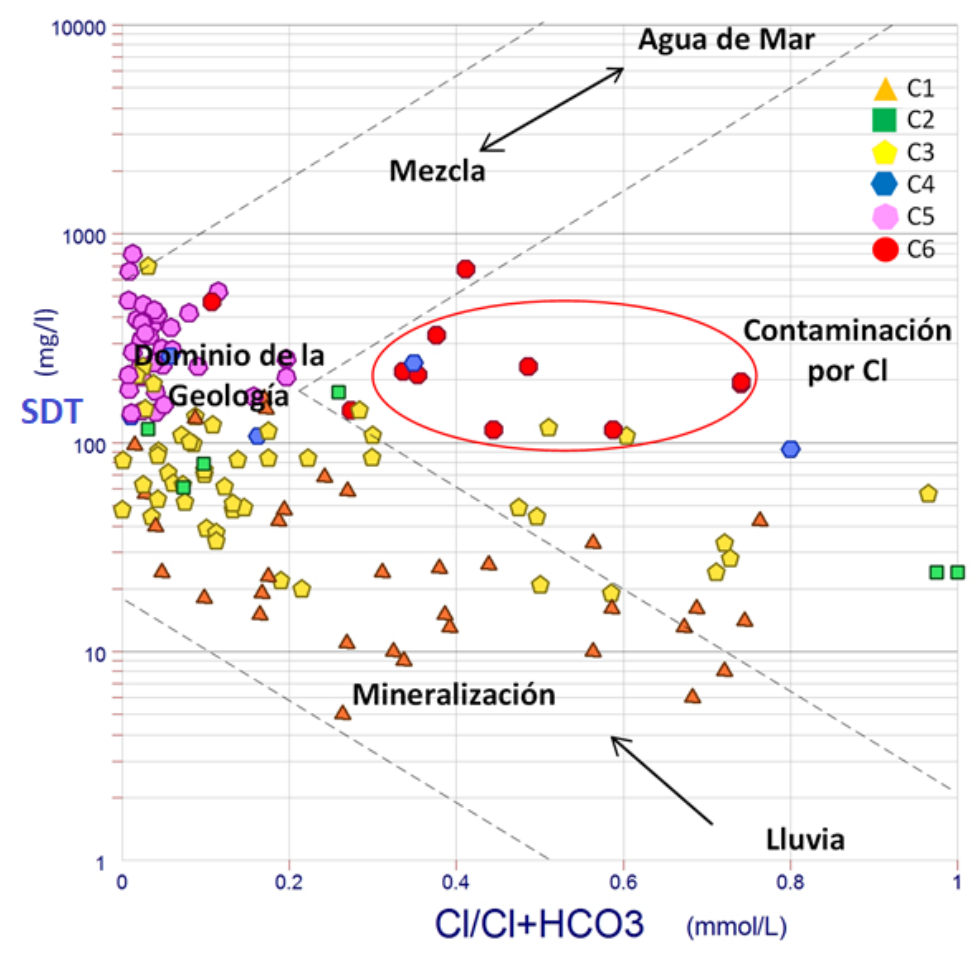

Figura 11 Diagrama de Gibbs con la proyección de los grupos obtenidos a partir de Análisis de Agrupamiento Jerárquico, se evidencia que las muestras de grupo 6 están fuera de los 3 dominios del diagrama, lo que podría ser un indicativo de contaminación por Clantropogénico producto de actividades petroleras. 
2014). Esta situación debe ser estudiada con mayor detalle, más allá que la concentración media (57.5 $\mathrm{mg} / \mathrm{L})$ y máxima $(123.6 \mathrm{mg} / \mathrm{L})$ del $\mathrm{Cl}^{-}$reportada estén por debajo del umbral establecido para agua potable $(250 \mathrm{mg} / \mathrm{L})$ en la normatividad nacional, es necesario determinar si hay una posible contaminación del agua subterránea por la actividad petrolera mediante monitoreos fisicoquímicos continuos.

\section{CONCLUSIONES}

En este estudio con base en la información compilada y analizada se identificaron los posibles procesos geoquímicos que determinan la composición química del agua subterránea ubicada en el SAVMM: Recarga- infiltración de agua meteórica, Disolución - interacción del agua con silicatos y carbonatos y Alteración Antropogénica - contaminación por el desarrollo de actividades industriales que se realizan en la región. Los resultados de este estudio demuestran claramente que el uso de métodos gráficos apoyados por la estadística mul-

a)

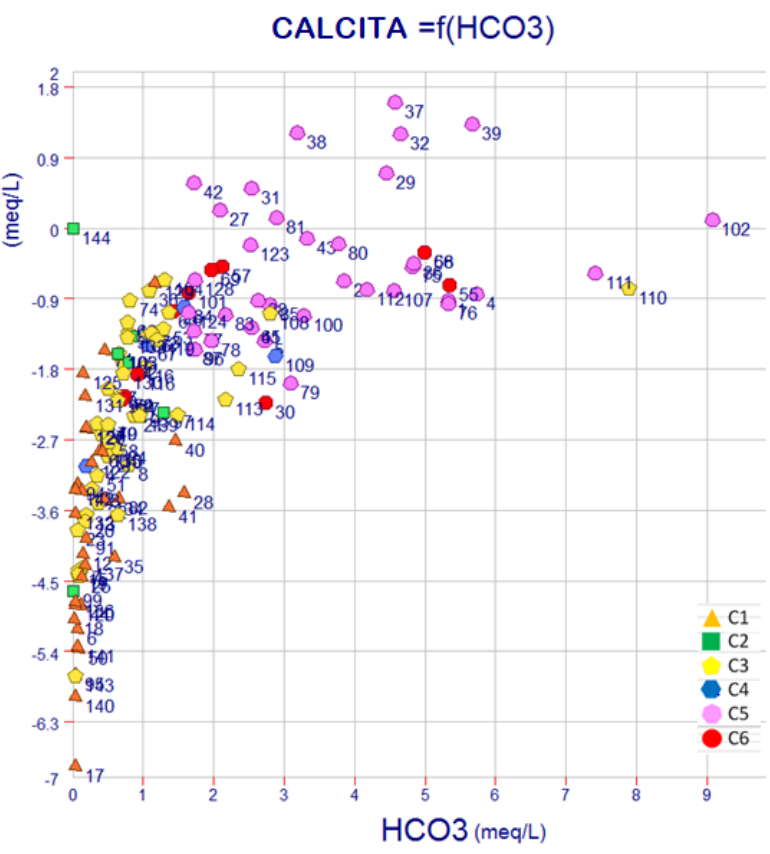

tivariada es de gran utilidad para clasificar un gran número de muestras basadas en sus parámetros químicos, con el fin de comprender con más detalle la hidrogeoquímica de un área de interés. Para el área de estudio, la facie hidrogeoquímica bicarbonatada cálcica es el tipo de agua dominante (51.4 $\%$ ), seguida de agua bicarbonatada sódica $(26.4 \%)$ y en menor proporción agua clorada sódica (15\%) y magnésica $(7.1 \%)$. Este comportamiento es consecuencia de la disolución de silicatos y carbonatos, y a su vez de las perturbaciones al sistema hidrogeológico resultado de las actividades antrópicas que se desarrollan en el área, específicamente la producción de hidrocarburos. Por medio del ACP se determinaron 3 componentes principales, los cuales explican el 59.4\% de la varianza total en el conjunto de datos. Se encontró que el primer componente principal está relacionado con disolución de silicatos y carbonatos, y el segundo y el tercero por una posible alteración antrópica. El AAJ permitió clasificar las 140 muestras en seis grupos de acuerdo con su similitud. La definición de los grupos sigue de cerca la clasificación por tipo de agua.

b)

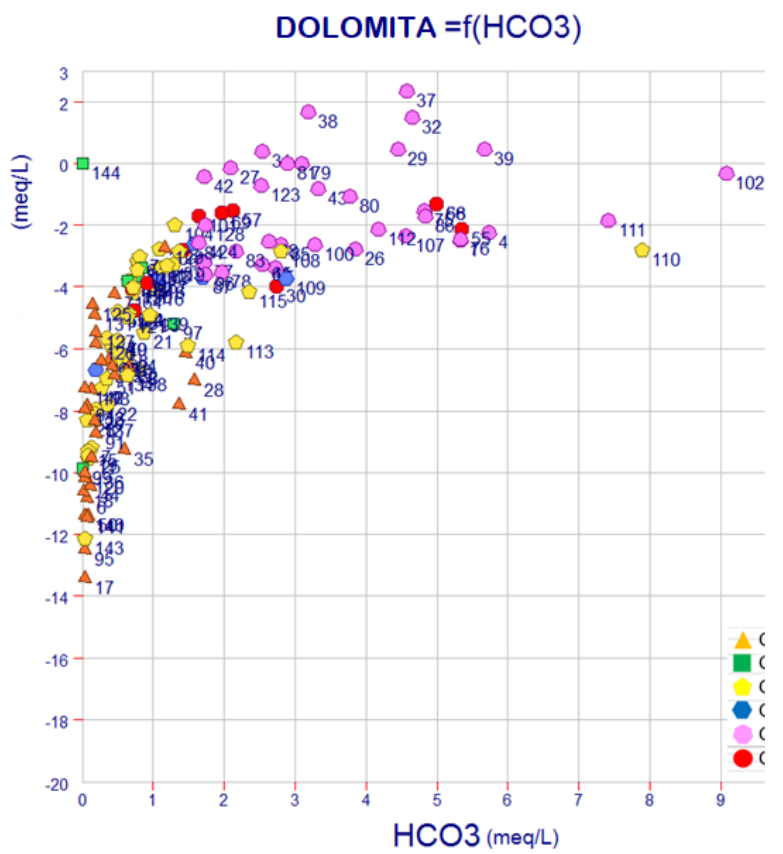


a)

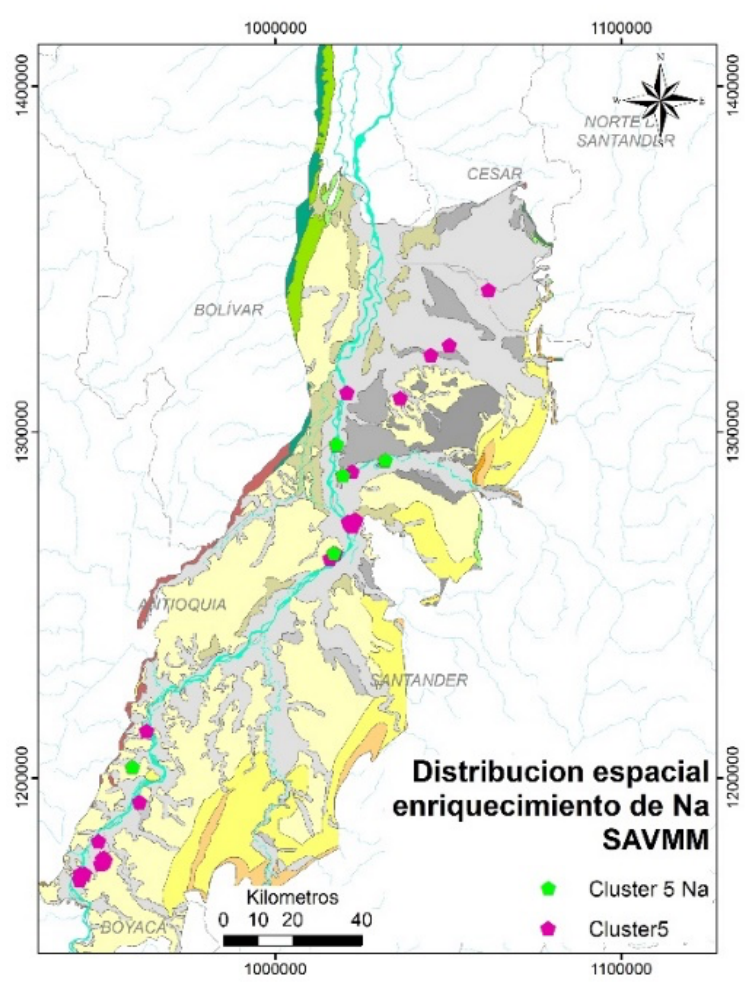

Escala 1:980.000 (modificado de IDEAM 2014) b)

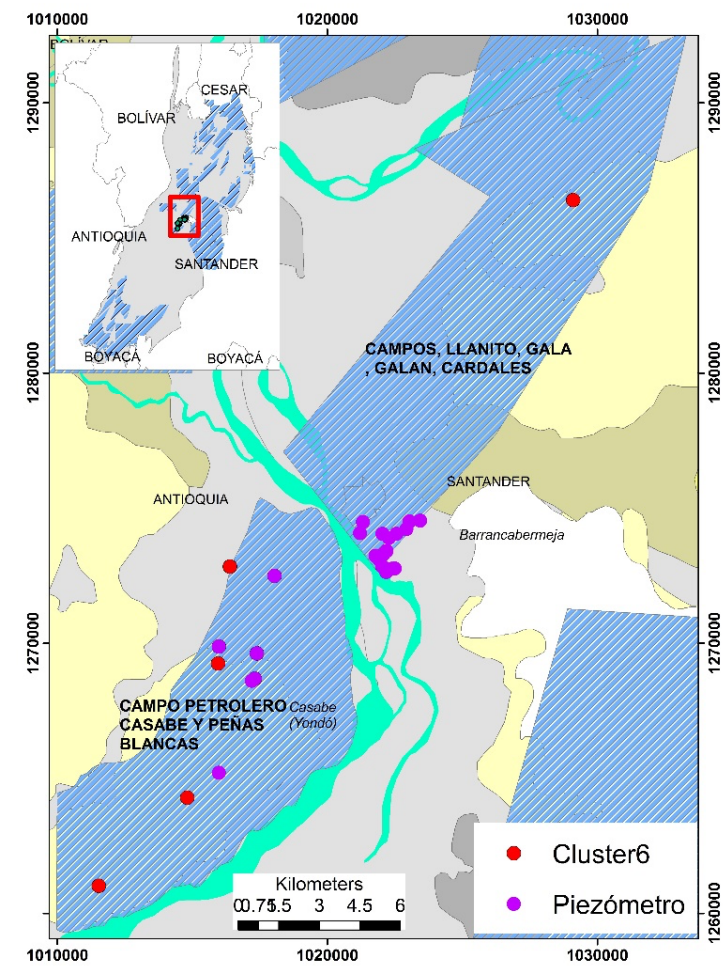

Escala 1:120.000 (modificado de ANLA 2015)

Figura 13 Distribución espacial de los grupos 5 y 6. a) Se resaltan las muestras con alto contenido de Na+ y b) Relación positiva en la ubicación de las muestras del grupo 6 y los piezómetros con registros altos de $\mathrm{Cl}$ - respecto a los campos petroleros Casabe y Llanito.

El grupo 1 corresponde al agua recién infiltrada en áreas de recarga, la cual es agua poco ionizada y corresponde a las muestras del tipo bicarbonatada cálcica. El grupo 5 representa el agua más ionizada, es de tipo bicarbonatada sódica y responde a agua subterránea más evolucionada dado que representa estratos más profundos, con mayor tiempo de residencia y tránsito a lo largo del área de estudio. Los grupos 2, 3 y 4 podrían corresponder a agua subterránea mixta o intermedia entre el grupo 1 y 5. Por otro lado, la geoquímica del grupo 6 es atípica, lo cual es explicado por un aporte de cloruro antropogénico.

\section{Agradecimientos}

Los autores quieren agradecer a los estudiantes que hicieron parte del programa nacional de semilleros de investigación, creación e innovación de la Universidad Nacional de Colombia 20132015 (Código: 27661), quienes con su dedicación ayudaron al procesamiento de la información y creación del "HidroVisor ASVAMM", a su vez a COLCIENCIAS y Universidad Nacional por el programa Jóvenes Investigadores e Innovadores No 617- (Código: 21579), quien otorgó una beca pasantía al autor principal Juan Pablo Malagón Navarro. También agradecemos a la Autoridad de Licencias Ambientales (ANLA) y al Instituto de Hidrología, Meteorología y Estudios Ambientales de Colombia (IDEAM) por facilitar el acceso a la información que fue utilizada en este estudio.

\section{Referencias}

Allen, R. G., Pereira,L. S., Raes,D., Smith,M., 1998, Crop evapotranspiration: Guidelines for computing crop water requirements. FAO 
Irrigation and drainage, paper 56. FAO, Rome, 300(9), D05109.http://www.fao. org/3/X0490E/X0490E00.htm

Agencia Nacional de Hidrocarburos (ANH), 2007, Colombian Sedimentary Basins: Colombia, ANH, $92 \mathrm{p}$.

Agencia Nacional de Hidrocarburos (ANH), 2012, Guenca Valle Medio del Magdalena: Integración geológica de la digitalización y análisis de núcleos: Colombia, ANH, 82 p.

Autoridad Nacional de Licencias Ambientales (ANLA), 2015, Por la cual se modifica el plan de manejo ambiental establecido mediante la resolución 0702 del 25 de Julio De 2002 y se toman otras determinaciones: Colombia,Ministerio de Ambiente y Desarrollo Sostenible, 198 p.

Arismendy Mejía, R., Salazar Villegas, J., Vélez Otálvaro, M., Caballero Acosta, H., 2003, Evaluación del potencial acuífero de los municipios de Puerto Berrío y Puerto Nare:Colombia, Escuela de Geociencias y Medio Ambiente, 19 p.

Blake, S., Henry, T., Murray, J., Flood, R., Muller, M. R., Jones, A. G., Rath, V., 2016, Compositional multivariate statistical analysis of thermal groundwater provenance: A hydrogeochemical case study from Ireland: Applied Geochemistry, 75, 171-188. https:// doi.org/10.1016/j.apgeochem.2016.05.008

Canavos, G.C., 1987, Probabilidad y estadística: México, McGraw-Hill.

Cañas, H., Pérez, O., Ruíz, D., Herrera, W., Watson, A., 2019, Modelo hidrogeológico conceptual Valle Medio del Magdalena. Planchas 108 y 119. Puerto Wilches, Barrancabermeja, Sabana de Torres y San Vicente de Chucurí: Colombia, Servicio Geológico Colombiano.

Cloutier, V., Lefebvre, R., Therrien, R., Savard, M. M., 2008, Multivariate statistical analysis of geochemical data as indicative of the hydrogeochemical evolution of groundwater in a sedimentary rock aquifer system: Journal of Hydrology, 353 (3-4), 294-313. https:// doi.org/10.1016/j.jhydrol.2008.02.015
Costa, D., Jesus, J., Branco, D., Danko, A., Fiúza, A., 2017, Extensive review of shale gas environmental impacts from scientific literature (2010-2015): Environmental Science and Pollution Research, 24(17), 14579-14594. https://doi.org/10.1007/ s1 1356-017-8970-0

Custodio, E.,Llamas, M.R., 2001, Hidrología Subterránea: España, Omega, 1224 p.

Chou, W. R., Fang, L. S., Wang, W. H., Tew, K. S., 2012, Environmental influence on coastal phytoplankton and zooplankton diversity: a multivariate statistical model analysis: Environmental Monitoring and Assessment, 184, 5679-5688. https://doi.org/10.1007/ s10661-011-2373-3

Darrah, T.H., Vengosh, A., Jackson, R.B., Warner, N.R., Poreda, R.J., 2014, Noble gases identify the mechanisms of fugitive gas contamination in drinking-water wells overlying the Marcellus and Barnett Shales: Proceedings of the National Academy of Sciences, 111(39), 14076-14081. https:// doi.org/10.1073/pnas.1322107111

Davis, J.C., Sampson, R.J., 2002, Statistics and data analysis in geology: New York, Wiley, $656 \mathrm{p}$.

De Porta, J., 1974, Lexique Stratigraphique International: Paris, Centre National de la Recherche Scientifique.

Departamento Nacional de Planeación (DNP), 2014, Bases del Plan Nacional de Desarrollo 2014-2018: Colombia, DNP, SITEAL, 784 p.

Environmental Protection Agency U.S. EPA, 2016, Hydraulic fracturing for oil and gas: Impacts from the hydraulic fracturing water cycle on drinking water resources in the United States: Office of Research and Development, Washington, DG. EPA/600/R-16/236Fa. 666p.

Everitt, B. S., Landau, S., Leese, M., Stahl, D., 2011, Cluster analysis, 5th ed.: New York, John Wiley \& Sons, 330 p. https://doi. org/10.1002/9780470977811 
Fagundo, J.R., González, H.P., 2005, Hidrogeoquímica: México, Centro Nacional de Medicina Natural y Tradicional (CENAMENT), 364 p.

Farnham, I.M., Johannesson, K.H., Singh, A.K., Hodge, V.F., Stetzenbach, K. J., 2003, Factor analytical approaches for evaluating groundwater trace element chemistry data: Analytica Chimica Acta, 490 (12), 123-138. https://doi.org/10.1016/ S0003-2670(03)00350-7

Farnham, I.M., Singh, A.K., Stetzenbach, K. J., Johannesson, K.H., 2002, Treatment of nondetects in multivariate analysis of groundwater geochemistry data: Chemometrics and Intelligent Laboratory Systems 60(1-2), 265-281. https://doi. org/10.1016/S0169-7439(01)00201-5

Freeze, R.A., Cherry, J.A., 1979, Groundwater: Canada, Prentice Hall, 604 p.

Ghesquière, O., Walter, J., Chesnaux, R., Rouleau, A., 2015, Scenarios of groundwater chemical evolution in a region of the Canadian Shield based on multivariate statistical analysis: Journal of Hydrology: Regional Studies, 4, 246-266. https://doi.org/10.1016/j. ejrh.2015.06.004

Gibbs, R.J., 1970, Mechanisms controlling world water chemistry: Science, 170, 1088-1090. https://doi.org/10.1126/ science.170.3962.1088

Giménez-Forcada, E., Vega-Alegre, M., TimónSánchez, S., 2017, Characterization of regional cold-hydrothermal inflows enriched in arsenic and associated trace-elements in the southern part of the Duero Basin (Spain), by multivariate statistical analysis: Science of the TotalEnvironment,593-594,211-226.https:// doi.org/10.1016/j.scitotenv.2017.03.07 1

Gong, F., Wang, B. T., Fung, Y. S., Chau, F. T., 2005, Chemometric characterization of the quality of the atmospheric environment in Hong Kong: Atmospheric Environment, 39(34), 6388-6397. https://doi.org/10.1016/j. atmosenv.2005.07.039
Güler, C., Thyne, G.D., McGray,J.E., Turner, K. A., 2002, Evaluation of graphical and multivariate statistical methods for classification of water chemistry data: Hydrogeology Journal, 10(4), 455-474. https://doi.org/10.1007/ s 10040-002-0196-6

Hounslow, A. W., 1995, Water quality data: Analysis and interpretation: New York, CRC Press, 416 p.

Instituto de Hidrología, Meteorología y Estudios Ambientales (IDEAM), 2013, Aguas Subterráneas en Colombia: una Visión General: Colombia, IDEAM, 284 p.

Instituto de Hidrología, Meteorología y Estudios Ambientales (IDEAM), 2014a, Estudio Nacional del Agua: Colombia, IDEAM, 496 p.

Instituto de Hidrología, Meteorología y Estudios Ambientales (IDEAM), 2014b, Sistemas Acuíferos de Colombia Anexo 4 ENA 2014, Fichas síntesis de sistemas acuíferos y aguas subterráneas frente a indicadores: Colombia, IDEAM, 77 p.

Instituto Nacional de Investigaciones GeológicoMineras (INGEOMINAS), 2002, Recopilación de Información Hidrogeológica para el Atlas de Aguas Subterráneas. Plancha 5-06. Informe Técnico: Colombia, INGEOMINAS, $51 \mathrm{p}$.

Instituto Nacional de Investigaciones GeológicoMineras (INGEOMINAS), 2003, Atlas de Aguas Subterráneas de Colombia en Escala 1:500000, Colombia.

James, G., Witten, D., Hastie, T., Tibshirani, R., 2016, An Introduction to statistical learning: with applications in R: New York, Springer, 426 p. https://doi. org/10.1007/978-1-4614-7138-7

Lang, S., 1990, Introducción al Algebra Lineal: Argentina, Addison-Wesley Iberoamericana, $365 \mathrm{p}$.

Lê, S., Josse, J., Husson, F., 2008, FactoMineR: An R Package for Multivariate Analysis: Journal of Statistical Software, 25(1), 1-18. https:// doi.org/10.18637/jss.v025.i01 
Lebart, L., Morineau, A., Piron, M.,1995, Statistique exploratoiremultidimensionnelle: París, Dunod, 456 p.

Matiatos, I., Alexopoulos, A., Godelitsas, A., 2014, Multivariate statistical analysis of the hydrogeochemical and isotopic composition of the groundwater resources in northeastern Peloponnesus (Greece): Science of The Total Environment, 476477, 577-590. https://doi.org/10.1016/j. scitotenv.2014.01.042

Mejía, R. O., 2008, El recurso hídrico en la jurisdicción de Corantioquia 1995-2007: Colombia, Corporación Autónoma de Antioquia (CORANTIOQUIA), 106 p.

Melloul, A., Collin, M., 1992, The 'principal components' statistical method as a complementary approach to geochemical methods in water quality factor identification; application to the Coastal Plain aquifer of Israel: Journal of Hydrology, 140(1-4), 49-73. https://doi. org/10.1016/0022-1694(92)90234-M

Mifflin, M.D., 1988, Region 5, Great Basin, in Back, W., Rosenshein, J.S., Seaber, P.R., (eds.), Hydrogeology: Boulder, CO: Geological Society of America, The Geology of North America, 69-86.

Montcoudiol, N., Molson, J., Lemieux, J.M., 2014, Groundwater geochemistry of the Outaouais Region (Québec, Canada): a regional-scale study: Hydrogeology Journal, 23(2), 377-396. https://doi.org/10.1007/ s10040-014-1190-5

Montgomery, R.D., Dietrich, W.E., 1992, Channel initiation and the problem of landscape scale: Science, 255, 826-830. https://doi. org/10.1126/science.255.5046.826

Mora, A., Rizzi, J., 2004, Regional prospectively evaluation. Reporte interno: Colombia, PETROBRAS.

Morales, L., 1958, General geology and oil occurrences of the Middle Magdalena Valley, Colombia. En Weeks, L.G. (Ed.), A Symposium Conducted by the American
Association of Petroleum Geologists: Habitat of Oil. AAPG, Tulsa, OK, 641-695.

Noshadi, M., Ghafourian, A., 2016, Groundwater quality analysis using multivariate statistical techniques (case study: Fars's province, Iran): Environmental Monitoring and Assessment, 188(7), 1-13.https://doi.org/10.1007/ s10661-016-5412-2

Osborn, S. G., Vengosh, A., Warner, N. R., Jackson, R. B., 2011, Methane contamination of drinking water accompanying gaswell drilling and hydraulic fracturing: Proceedings of the National Academy of Sciences, 108(20), 8172-8176. https://doi. org/10.1073/pnas. 1100682108

Peeters, L., 2014, A background color scheme for piper plots to spatially visualize hydrochemical patterns: Ground Water 52(1), 2-6. https:// doi.org/10.1111/gwat.12118

Piper, A. M., 1944, A graphic procedure in the geochemical interpretation of wateranalyses: American Geophysical Union, 26(6), 914-928. http://dx.doi.org/10.1029/ TR025i006p00914

R Development Core Team, 2011, A Language and Environment for Statistical Computing: USA, R Foundation for Statistical Computing. Servicio Geológico de los Estados Unidos, (USGS), 2018, PHREEQG Versión 3. Estados Unidos.

Simler, R., 2013, DIAGRAMMES: Logiciel d'hydrochimie multilangage. Software en distribution libre.

Steinhorst, K. R., Williams, R. E., 1985, Discrimination of groundwater sources using cluster analysis, MANOVA, Canonical Analysis and Discriminant Analysis: Water Resources Research 21(8), 1149-1156. https://doi.org/10.1029/ WR021i008p01149

Stiff, H. A., 1951, The Interpretation of Chemical Water Analysis by Means of Patterns: Journal of Petroleum Technology, 3(10), 1-15. https://doi.org/10.2118/951376-G

Taborda, B., 1965, Anual field conference Guidebook to the geology of the Mares 
Concession: Bogotá, Colombian Society of Petroleum Geologist and Geophysicists, 44 p.

Tikhomirov, V.V., 2016, Hydrogeochemistry fundamentals and advances: New York, Wiley, $498 \mathrm{p}$.

Villegas, P., Paredes, V., Betancur, T., Ribeiro, L., 2013, Assessing the hydrochemistry of the Urabá Aquifer, Colombia by principal component analysis: Journal of Geochemical Exploration 134, 120-129. https://doi. org/10.1016/j.gexplo.2013.08.011

Ward, D., Goldsmith, R., Cruz, B., Jaramillo, C., Restrepo, H., 1973, Geología de los Cuadrángulos H-12, Bucaramanga y H-13, Pamplona, Departamento de Santander: Boletín Geológico, 21(1-3), 1-132. 